Original Articles

\title{
Exploiting risk-reward structures in decision making under uncertainty
}

\author{
Christina Leuker*, Thorsten Pachur, Ralph Hertwig, Timothy J. Pleskac \\ Center for Adaptive Rationality, Max Planck Institute for Human Development, Berlin, Germany
}

\section{A R T I C L E I N F O}

\section{Keywords:}

Decisions under uncertainty

Adaptive cognition

Risk and reward

Incidental learning

Ecological rationality

\begin{abstract}
A B S T R A C T
People often have to make decisions under uncertainty-that is, in situations where the probabilities of obtaining a payoff are unknown or at least difficult to ascertain. One solution to this problem is to infer the probability from the magnitude of the potential payoff and thus exploit the inverse relationship between payoffs and probabilities that occurs in many domains in the environment. Here, we investigated how the mind may implement such a solution: (1) Do people learn about risk-reward relationships from the environment-and if so, how? (2) How do learned risk-reward relationships impact preferences in decision-making under uncertainty? Across three experiments $(N=352)$, we found that participants can learn risk-reward relationships from being exposed to choice environments with a negative, positive, or uncorrelated risk-reward relationship. They were able to learn the associations both from gambles with explicitly stated payoffs and probabilities (Experiments 1 \& 2) and from gambles about epistemic events (Experiment 3). In subsequent decisions under uncertainty, participants often exploited the learned association by inferring probabilities from the magnitudes of the payoffs. This inference systematically influenced their preferences under uncertainty: Participants who had been exposed to a negative risk-reward relationship tended to prefer the uncertain option over a smaller sure option for low payoffs, but not for high payoffs. This pattern reversed in the positive condition and disappeared in the uncorrelated condition. This adaptive change in preferences is consistent with the use of the risk-reward heuristic.
\end{abstract}

\section{Introduction}

In March 2016, James Stocklas won \$291 million in the Florida Powerball lottery. Most people know that winning such a huge jackpot is a pretty unlikely event. Now consider his brother, Bob Stocklas. Bob bought a ticket for the same lottery at the same time as James and won just $\$ 7$ (Newsome, 2016). Most people know that winning this kind of sum is far more likely than winning the jackpot. And, of course, most people are also painfully aware that not winning anything at all is much more likely than either of these events. While this story illustrates the strange vicissitudes of fortune, for our purposes it also illustrates just how comfortable people are with estimating the probability of winning from payoff magnitudes alone. How do people "know" how to estimate the chances of winning the lottery? Why do they associate the highest payoff with the lowest probability? Here, we argue that the key to understanding how the mind generates such estimates lies not within the mind alone, but how the mind is adapted to its environmental context (Anderson, 1991; Gibson, 1979; Gigerenzer, Hertwig, \& Pachur, 2011; Marr, 1982; Perkovic \& Orquin, 2017; Shepard, 1987; Simon, 1956; Stewart, Chater, \& Brown, 2006).

Beyond the lottery, risks and rewards, or payoffs and probabilities, are linked in many choice environments. Across choice environments, probably the most frequent and recurrent link between them is an inverse relationship: The higher rewards that we desire are unlikely to be obtained (Pleskac \& Hertwig, 2014). However, the strength of the relationship also varies across different domains. Monetary gambles in casinos, for instance, show a near perfect (though biased) inverse relationship between payoffs and probabilities. In other domains, such as where to submit a scientific manuscript (trading off impact factor against acceptance rate), the risk-reward relationship is less strong. Moreover, a risk-reward relationship is not always given. For instance, no relationship between risk and reward is to be expected in newly forming markets, that have not yet reached an equilibrium (Pleskac \& Hertwig, 2014).

After identifying the ecological structures in which the mind usually operates, one can try to establish how the mind comes to terms with those ecological structures (Brunswik \& Kamiya, 1953; Simon, 1956): Risk-reward structures can be exploited in decisions under uncertainty-where people have to choose between options whose payoffs are known but probabilities are not (Knight, 1921; Luce \& Raiffa, 1957; Wakker, 2010). Pleskac and Hertwig (2014) offered participants a gamble that gave them a chance to win $\$ x$ at the cost of $\$ 2$, and asked

\footnotetext{
* Corresponding author at: Max Planck Institute for Human Development, Center for Adaptive Rationality, Lentzeallee 94, 14195 Berlin, Germany.

E-mail address: leuker@mpib-berlin.mpg.de (C. Leuker).
} 


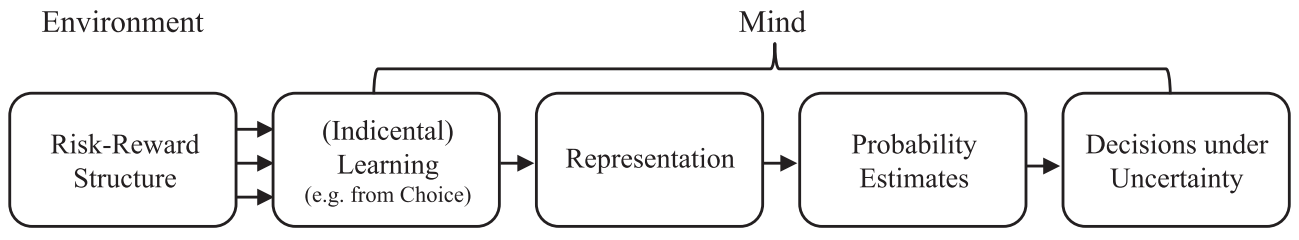

Fig. 1. Summary of the assumed relationships among risk-reward structures in the world and how they ultimately shape preferences under uncertainty.

them to estimate the probability of winning $\$ x$. Different participants were asked to consider different magnitudes of $x$. As the magnitude of the potential payoff increased, the estimated probabilities of winning decreased. That is, participants inferred the probabilities to be inversely related to the magnitude of the payoff. Moreover, the estimates ultimately influenced what participants chose.

Inferring a probability from the magnitude of the potential payoff might be an adaptive solution to decision-making under uncertainty-a solution that Pleskac and Hertwig (2014) refer to as the risk-reward heuristic. Here, we investigate two of its requirements: First, the mind has to be sufficiently sensitive to the relationship between the key variables in an environment (Brunswik, 1955; Gigerenzer, Hoffrage, \& Kleinbolting, 1991; Gibson, 1979; Marr, 1982; Simon, 1956; Stewart et al., 2006) or even mirror the relationship from the environment (Anderson \& Schooler, 1991; Shepard, 1967, 1987). Second, people should be willing to harness the structure flexibly, as the ecological regularity varies across environments (Todd \& Gigerenzer, 2007). That is, there should be a link between the estimates people give and an environments' risk-reward structure. This link also means that, for instance, people should withhold from estimating a high payoff to be unlikely if appropriate (e.g., in a newly forming market). This argument can be developed further: Payoffs and (subjective) probabilities determine the value of an option, and ultimately choice. Therefore, different risk-reward environments should not only affect the estimates themselves but also decisions under uncertainty.

Fig. 1 provides an overview of the assumed relationships between risk-reward structures and choice that we take in this paper. Next, we develop our hypotheses in more detail, before reporting three experiments to test them.

\subsection{How can people learn risk-reward structures?}

In most domains, people are not explicitly told about the presence and/or direction of a risk-reward relationship. They also often do not have the luxury to learn about the relationship from explicit feedback. In this case, a risk-reward relationship would need to be acquired as people go about their primary objective when making decisions. In other words, the risk-reward relationship would seem to be learned in an unsupervised manner (without corrective feedback; Love, 2002), and incidentally (when learning is not the primary objective; Brooks, 1978; Dulany, Carlson, \& Dewey, 1984; Nelson, 1984; Ward \& Scott, 1987; Wattenmaker, 1991; Whittlesea, 1987). ${ }^{1}$

Prior research suggests that via such incidental learning, people can be remarkably well attuned to statistical structures of their choice environments. For instance, they are quite good at learning the frequencies of events, even when that is not their central task (Hasher \& Zacks, 1979; Hasher, Zacks, Rose, \& Sanft, 1987; Zacks, 2002). People also appear to encode the prices of goods and to use those prices later to evaluate the subjective worth of new values (Brown, Gardner, Oswald, \& Qian, 2008; Olivola \& Sagara, 2009; Stewart et al., 2006; Ungemach, Stewart, \& Reimers, 2011), or use marginal distributions of either

\footnotetext{
${ }^{1}$ One might also classify this as a case of implicit learning (see, e.g., Cleeremans, Destrebecqz, \& Boyer, 1998; Frensch \& Rünger, 2003; Reber, 1967; Reber, 1989; Seger, 1994; Shanks \& St. John, 1994). However, a typical condition for implicit learning is that individuals lack awareness of what is learned. We are thus hesitant to use this concept, as it seems that people are aware of the risk-reward relationship (Pleskac \& Hertwig, 2014).
}

payoffs or probabilities in subjective evaluations thereof (Stewart, Reimers, \& Harris, 2015; Walasek \& Stewart, 2015). However, the risk-reward relationship is different from encoding and using (marginal) distributions of probabilities/frequencies and payoffs in that it requires people to learn a statistical regularity between probabilities and payoffs (i.e., a joint distribution). It is well known that people can learn associations between two variables (e.g., between a cue and a criterion, see Cooksey, 1996), and sometimes fairly quickly (Kareev, 2000; but see Anderson, Doherty, Berg, \& Friedrich, 2005). It is not known whether these findings extend to preferential choice in general; and (maybe even more importantly) to what extent people can learn that there is no correlation in their environment, as people may be biased to detecting structures where there are none (Langer, 1975; Olivola \& Oppenheimer, 2008).

To test people's ability to learn a risk-reward relationship in an unsupervised, incidental manner, we created a learning phase in which participants encountered gambles where payoffs and probabilities were negatively correlated, positively correlated, or uncorrelated. Across experiments, we tested participants' ability to learn from different types of gambles: In Experiments 1 and 2, participants were asked to evaluate risky monetary gambles of the form " $p$ chance of winning $x$, otherwise nothing." In Experiment 3, we examined to what extent participants learned different risk-reward structures from epistemic events when the probabilities were subjective (see also Tversky \& Fox, 1995; Tversky \& Wakker, 1995). Across experiments we also examined how different response types impacted learning with participants either choosing between gambles (Experiment 1) or stating the price for which they would be willing to sell individual gambles for (Experiments 2 and 3).

Finally, we examined in what form the risk-reward relationship is represented. In Experiments 1 and 2, we asked participants if they recognized specific gambles from the earlier learning phase. In so doing, we tested whether the risk-reward structure was learned as a "risk-reward rule" or via memory of specific gamble exemplars (Erickson \& Kruschke, 1998): If it was learned via exemplars, participants should be able to recognize specific gambles from the learning phase (but not similarly structured foils).

\section{2. (How) are different risk-reward structures exploited in decisions under uncertainty?}

If risk-reward structures are used in decisions under uncertainty to infer the values of missing probabilities, then this can give rise to environment-dependent preferences. To see this, consider an environment with a negative risk-reward relationship where high payoffs are unlikely. Someone exposed to this environment is offered a choice between an uncertain gamble with a very high payoff or a smaller, say half-as-large, certain payoff. He or she should prefer the certain payoff (i.e., the sure thing). This is because, according to the risk-reward heuristic, he or she will estimate the chances of obtaining the high uncertain payoff to be quite low and as a result the sure outcome $(x)$ will outweigh the uncertain outcome $(y)$ multiplied by its inferred probability $\left(x>p_{\text {inferred }} \times y\right)$. The decisions of someone who has learned that risks and rewards are positively related can be expected to show the opposite pattern. Lastly, someone who has learned that risks and rewards are uncorrelated can be expected to make decisions as if the probability estimates assigned to events were independent of their payoffs. He or she may adhere to the principle of indifference, assign a 
Table 1

Overview of experiments and conditions.

\begin{tabular}{|c|c|c|c|c|}
\hline \multirow[t]{2}{*}{ Experiment } & \multicolumn{2}{|c|}{ Learning phase } & \multirow[t]{2}{*}{ Test phase (condition-independent) } & \multirow[t]{2}{*}{ Aim of experiment } \\
\hline & Task & Conditions & & \\
\hline 1 & Choice & $\begin{array}{c}\text { Negative } \\
\text { Uncorrelated }\end{array}$ & $\begin{array}{l}\text { Decisions under uncertainty } \\
\text { Payoff-probability estimation } \\
\text { Recognition }\end{array}$ & $\begin{array}{l}\text { Incidental learning of risk-reward structures } \\
\text { Influence on decisions under risk and uncertainty }\end{array}$ \\
\hline 2 & WTS & $\begin{array}{l}\text { Negative } \\
\text { Positive } \\
\text { Uncorrelated }\end{array}$ & $\begin{array}{l}\text { Decisions under uncertainty } \\
\text { Payoff-probability estimation } \\
\text { Recognition } \\
\text { Probability-payoff estimation }\end{array}$ & $\begin{array}{l}\text { Incidental learning of a positive risk-reward structure } \\
\text { Influence of type of learning phase task } \\
\text { Influence on decisions under uncertainty }\end{array}$ \\
\hline 3 & WTS & $\begin{array}{l}\text { Negative risk } \\
\text { Positive risk } \\
\text { Negative uncertain } \\
\text { Positive uncertain }\end{array}$ & $\begin{array}{l}\text { Decisions under uncertainty } \\
\text { Subjective probability estimation } \\
\text { Payoff-probability estimation }\end{array}$ & $\begin{array}{l}\text { Gambles with epistemic events } \\
\text { Incidental learning under risk vs. uncertainty } \\
\text { Influence on beliefs about events }\end{array}$ \\
\hline
\end{tabular}

Note. Learning phase stimuli were condition-dependent. All test phase tasks were condition-independent. WTS: Willingness to sell.

probability of .5 to each outcome (Fox \& Clemen, 2005; Fox \& Rottenstreich, 2003), and choose the uncertain alternative equally often across payoff magnitudes.

Alternatively, preferences might be stable and simply revealed as people are asked to make decisions (i.e., revealed preference theory) (McFadden, Machina, \& Baron, 1999). A cornerstone of revealed preference theory is the principle of description invariance where the preferences and beliefs should be invariant to the description of the event (Tversky \& Kahneman, 1986; Tversky, Sattath, \& Slovic, 1988): Given a choice between an uncertain gamble and a half-as-large certain payoff, inferred probabilities and ultimately preferences should not depend on the magnitudes of the payoffs (e.g., 1 for sure vs. 2 with an unknown probability should elicit the same preference as 1000 for sure vs. 2000 with an unknown probability). Consequently, the risk-reward environment should not impact preferences at all.

A last prediction on how risk-reward structures might impact how people deal with missing probability information in decisions under uncertainty can be derived from research on the desirability or optimism bias (Bar-Hillel \& Budescu, 1995; Edwards, 1962; Irwin, 1953; Krizan \& Windschitl, 2007; Sharot, 2011; Windschitl, Smith, Rose, \& Krizan, 2010). Here, as payoffs become more desirable, they (or the event with which they are associated) are perceived as more likely. This prediction could either hold irrespective of the statistical relationship between risk and reward or contribute to people's environment-dependent inferences. The affect heuristic, according to which more positive overall affect towards high payoffs can mitigate perceived risk (Pachur, Hertwig, \& Steinmann, 2012; Slovic \& Peters, 2006; Slovic, Finucane, Peters, \& MacGregor, 2004), would yield a similar prediction. That is, both the optimism bias and the affect heuristic may support the belief that-probably within limits—high payoffs are by no means unlikely.

\subsection{Overview of experiments}

We conducted three experiments, each consisting of a conditiondependent learning phase and a test phase (Table 1). In Experiments 1 and 2, learning environments consisted of gambles of the form " $p$ chance of winning $x$, otherwise nothing." In Experiment 3, the gambles were about an epistemic event, namely, whether the maximum temperature in Berlin on a particular day in 2011 fell within a given range. This design allowed us to examine how well participants learned risk-reward structures from gambles in which the probabilities were not explicitly stated.

In all three experiments, environments were constructed such that across the gambles probabilities and payoffs were either negatively correlated, positively correlated, or uncorrelated. Importantly, participants were neither informed about the risk-reward structures nor asked to attend to them; instead they merely experienced the structure by evaluating monetary gambles. After the learning phase, we tested how exposure to different risk-reward environments impacted participants' preferences among uncertain options. Participants then completed payoff-probability estimation tasks, which we used to test whether they had learned the risk-reward structure. In Experiments 1 and 2, they also completed a gamble recognition task that tested whether the structure was learned via memory of specific exemplars of gambles or as a rule.

\section{Experiment 1: Do people learn negative vs. uncorrelated risk-reward environments and exploit them in decisions under uncertainty?}

Our first experiment had an exploratory focus. We designed it to examine how the risk-reward structure impacts decision making under both risk (probabilities given) and uncertainty (probabilities missing). To this end, we exposed participants to different risk-reward environments, asking them to choose between two nondominating gambles of the form " $p$ chance of winning $x$, otherwise nothing." Between participants, the gambles were selected from one of two environments. In the negative environment, there was a negative (linear) relationship between payoffs and probabilities across all possible gambles. In the uncorrelated environment, payoffs and probabilities were randomly paired. We hypothesized that participants would learn about the risk-reward structures as a consequence of their primary task, which was to choose the alternative they preferred.

To examine how the different risk-reward structures impacted decision making under risk, about halfway through this learning phase we included gambles common to both conditions, including eight gambles designed to examine the certainty effect (Fig. 2). However, we found very little differences between the conditions with respect to choices under risk. For instance, we found the certainty effect in both conditions. As our article is focused on decisions under uncertainty, these analyses on decisions under risk are reported in the Supplementary Material (see also Leuker, Pachur, Hertwig, \& Pleskac, 2018; Leuker, Pleskac, Pachur, \& Hertwig, 2017).

After the learning phase, participants completed three tasks that were identical across both conditions (test phase) (see also Table 1). The first task was the decision making under uncertainty task designed to test the environment-dependent preference prediction of the risk-reward heuristic. We then tested to what extent participants learned the respective risk-reward structure by explicitly asking them to estimate probabilities when presented with new payoffs. Finally, we administered a recognition task to investigate whether participants remembered specific gambles (exemplars) or whether they had extracted a "risk-reward rule" from the learning phase. 


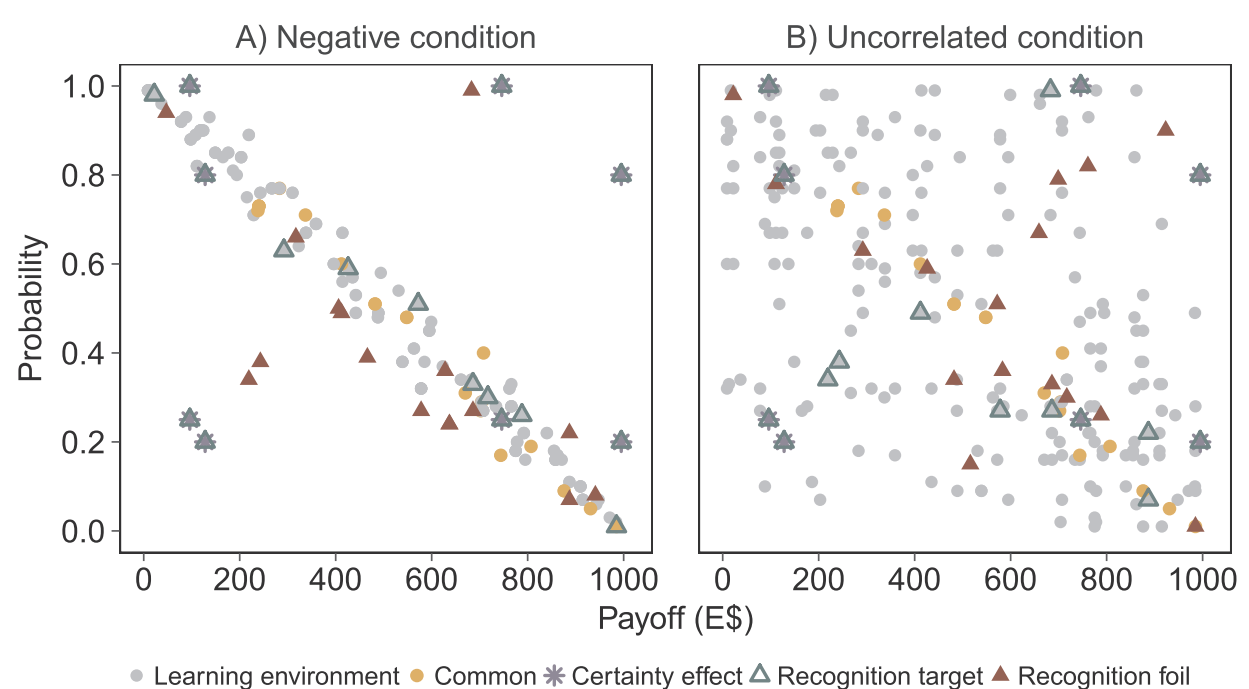

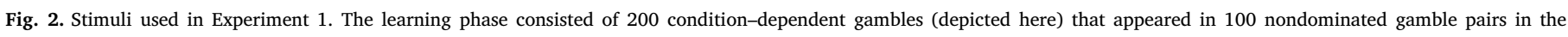

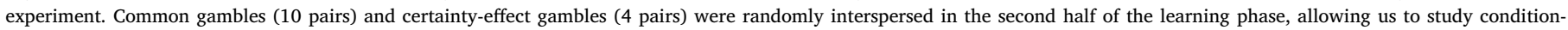
dependent changes in decisions under risk. Dominated options not depicted.

\subsection{Method}

\subsubsection{Participants}

We set a target sample size of 60 participants. In total, the sample comprised 62 adults ( 32 females, mean age $=25.6, S D=3.4$, proportion students $=.93$ ) from the participant pool maintained at the Max Planck Institute for Human Development (32 in the negative condition, 30 in the uncorrelated condition). ${ }^{2}$ All experiments were approved by the IRB of the Max Planck Institute for Human Development. Participants gave signed informed consent prior to the experiment; they were paid a fixed rate of $10 € / \mathrm{h}$ plus a bonus contingent on their choices.

\subsubsection{Decisions under risk (learning phase)}

During the learning phase, participants repeatedly chose between two monetary gambles of the form " $p$ chance of winning $x$, otherwise nothing." All payoffs across all three conditions were expressed using an experimental currency, E\$. We did this with the goal of minimizing the impact of outside norms associated with specific currencies on the experiments. Each individual gamble was selected from either a negative or uncorrelated risk-reward environment (Fig. 2). The experiment's negative risk-reward environment consisted of 200 gambles (but 100 nondominated gamble pairs) that followed a negative linear (though slightly noisy) risk-reward environment. Precise details on how the gambles were created and paired can be found in the Supplementary Materials (plus code on the OSF).

For the uncorrelated condition, we took the 200 gambles (100 gamble pairs) used in the negative condition, but now randomly linked probabilities and payoffs. If any of the gamble pairs had stochastically dominated options (i.e., $p_{A}>p_{B}$ and $x_{A}>x_{B}$ ), we switched the probabilities of gambles $\mathrm{A}$ and $\mathrm{B}$. We did this to maintain the marginal distributions of payoffs and probabilities across both conditions (see Stewart et al., 2006, 2015).

In both conditions, we included five dominated options that we used as 'catch trials' to identify participants who did not pay attention. In addition, 14 identical gamble pairs appeared in both conditions. Ten of these pairs were created based on the procedure for the negatively correlated risk-reward environment. The other four pairs were designed to examine the certainty effect (see Supplementary Materials).

\footnotetext{
${ }^{2}$ Ten other participants also completed the experiment, but a coding error in the computerized experiment corrupted their data.
}

Across participants, we randomized the positions of the gambles on screen, and counterbalanced the location of payoffs and probabilities (top/bottom).

\subsubsection{Decisions under uncertainty (test phase)}

We drew 20 random payoffs (range E\$1-1000) and gave them a probability of "?". Each uncertain payoff $y$ was then matched with a half-as-large certain option (probability 100\%). For example, one pair of gambles asked participants to chose between a $100 \%$ chance of winning $\mathrm{E} \$ 50$ and a “?" chance of winning $\mathrm{E} \$ 100$. We also included 20 filler trials, in which the certain payoffs were also smaller, but created using different fractions of the uncertain payoffs. We did this to ensure that participants attended to payoffs, probabilities and uncertainty in each trial. The location of the uncertain option was counterbalanced across participants. The location of the payoffs and probabilities (top/ bottom) matched the location used during the learning phase.

\subsubsection{Payoff-probability estimation task (test phase)}

To test the extent to which (individual) participants had learned about condition-dependent risk-reward relationships, we drew 10 random payoffs (range E\$1-1000), and later asked participants for their estimates of the associated probabilities.

\subsubsection{Recognition (test phase)}

Finally, to test whether participants recognized specific gambles that did not fit the risk-reward structure of a condition (gambles "off" the slope, see Fig. 2), we asked participants whether they recognized (yes or no) gambles from the learning phase. The recognition task included (1) certainty-effect gambles as a particular case of exemplars that people may recall particularly well, (2) eight environment gambles from the learning phase as a subsample of exemplars that people may have encoded during learning, (3) eight environment gambles that did not appear in the learning phase (but matched the gamble structure of the condition), and (4) eight environment gambles that appeared in the other condition (thus did not match the gamble structure of the condition). This resulted in 32 cued-recognition trials (16 targets, 16 foils; see triangles in Fig. 2).

\subsubsection{Procedures}

Participants were randomly assigned to either the negative or the uncorrelated condition. Participants were told that they would be asked to make a series of choices between monetary gambles in the first part 

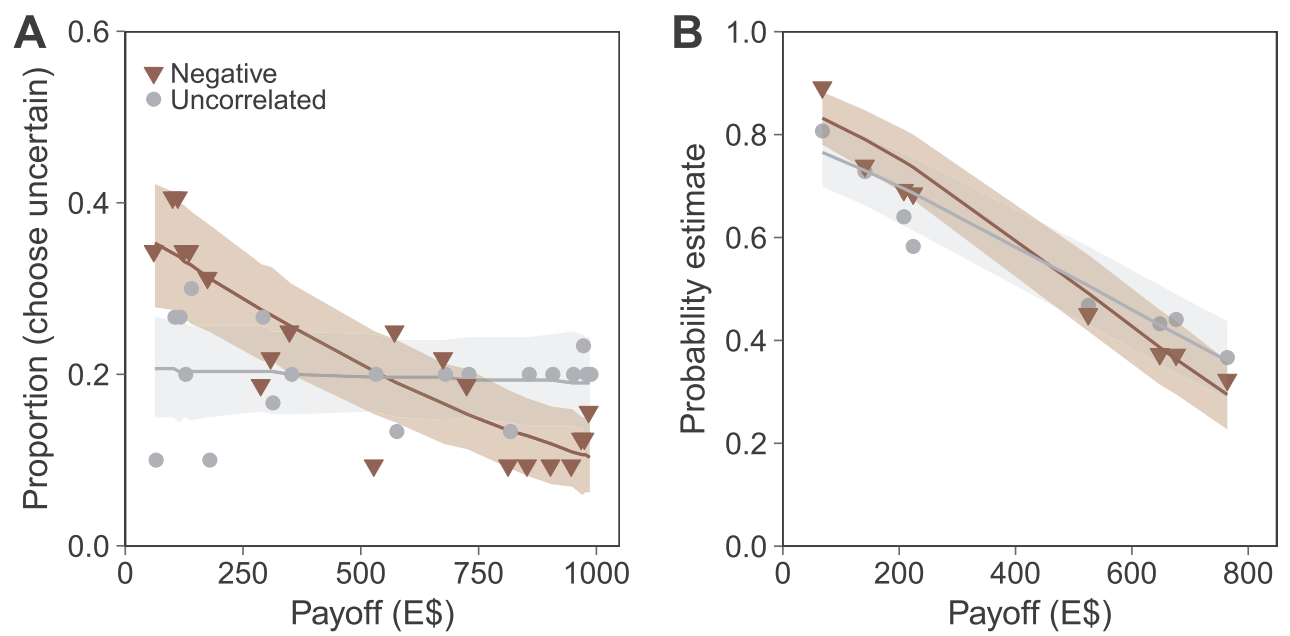

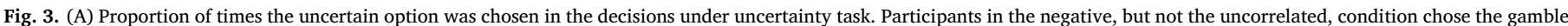

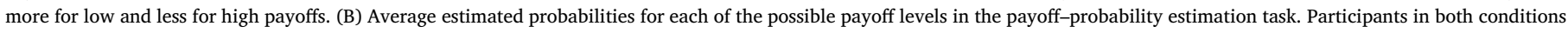

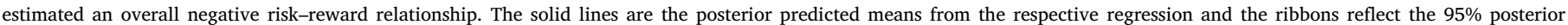
predictive distribution.

of the experiment, and that there would then be some additional questions. All experiments were coded in PsychoPy (Peirce, 2007). Screenshots of all experiments can be found in the Supplementary Materials.

In the learning phase, participants saw a fixation cross (for $500 \mathrm{~ms}$ ) before making a choice between two gambles. The chosen option was highlighted for $500 \mathrm{~ms}$ (by a red rectangle around the gamble), but participants did not receive any feedback about their actual payoff until the very end of the experiment. Participants took self-paced breaks after blocks of 30 trials. Gambles were presented in random order. The gambles common to both conditions were randomly interspersed after 50 condition-dependent learning trials.

To link the test phase with the learning phase, we told participants that they would see gambles that were structured similarly to the gambles they had experienced previously, and asked them to think back to these gambles when completing the task given. The order of tasks in the test phase was counterbalanced, with one constraint: Participants always completed the probability estimation task after the decision under uncertainty trials to minimize experimental demand effects in the choice task (i.e., prompting participants to infer probabilities from payoff magnitudes).

At the end of the experiment, we played out the chosen option of 20 randomly drawn trials of the learning phase. Bonuses (between $1.92 €$ and $7.74 €$, with $\mathrm{E} \$ 1000=1 €$ ) were added to the regular payment.

\subsubsection{Analyses}

We used a Bayesian approach to data analysis (Kruschke, 2014). Specifically, we applied Bayesian Generalized Linear Mixed Models using Stan in $\mathrm{R}$ for regression analyses with the rstanarm package (Stan Development Team, 2016). Unless otherwise noted, we entered participant as a grouping factor to account for individual variation beyond condition-dependent effects. Choice data were analyzed using logistic regressions; estimation data (restricted between $[0,1]$ ) were modeled after response data had been transformed to a logit scale. When plotting the posterior-predictive fits of the statistical model, we back-transformed the estimates using the inverse logit. When analyzing probability estimates, we analyzed both the estimates and the logit transformations of the estimates. For ease of interpretation, we report the results from the analyses using untransformed estimates (both analyses resulted in qualitatively identical conclusions).

We ran three chains using a Markov Chain Monte Carlo sampler to draw from posterior distributions of parameters. Depending on model complexity, we ran 10,000-30,000 samples per chain (to ensure an effective sample size of $>10,000$ for each regressor) and set a burn-in of 500 samples. We investigated (convergence of) our posteriors through visual inspection and the Gelman-Rubin statistic (Gelman \& Rubin, 1992). In general, we report the mean of the posterior distribution of the parameter or statistic of interest and two-sided $95 \%$ equal-tail credible intervals (CI) around each value. Our focus is on estimating the effects of particular conditions and our analyses reflect this goal; in comparing the conditions, however, the crucial issue was whether the credible values included 0 or not.

\subsection{Results}

\subsubsection{Decisions under risk (learning phase)}

We examined choices in the learning phase to see how different risk-reward environments impacted decision making under risk. Four participants chose a stochastically dominated option once, all in the negative condition. The differences in expected values in these trials were small $\left(E V_{\text {abs }}=\mathrm{E} \$ 60\right.$ and $\left.\mathrm{E} \$ 5\right), E V_{\%}=6.0 \%$ and $0.5 \%$ and thus potentially hard to detect. We therefore included these participants' data in further analyses.

Choices between gambles were consistent with standard theories of choice: Participants chose the higher expected value gamble in $79 \%$ of all trials $(O R=5.38, b=1.68, \mathrm{CI}=[1.48,1.89])$, and this preference did not differ between the environments $\left(O R=.82, b_{\text {negative }}=-0.19, \mathrm{CI}=\right.$ $[-0.68,0.30])$. Moreover, larger EV differences leading to more EVmaximizing choices $\left(b_{\mathrm{EV}}=.008, \mathrm{CI}=[.007, .009]\right.$, in a logistic regression with EV differences, higher EV, and condition as predictors). This pattern of results persisted when we compared choices in the subset of common gambles only. In addition, there were no differences in participants' subjective evaluations of payoffs and probabilities as modeled by prospect theory (Tversky \& Kahneman, 1992) (see Supplementary Material for further details and other hypotheses we tested). In sum, we did not find evidence that manipulated risk-reward structures systematically impacted decision-making during the learning phase.

\subsubsection{Decisions under uncertainty (test phase)}

Did payoff levels shape preferences depending on the risk-reward structure experienced? In decisions under uncertainty, participants in both conditions preferred the sure option over the uncertain one $\left(M_{\text {uncertain }}=.21, b=1.89, \mathrm{CI}=[1.44,2.40]\right)$. However, as predicted, the strength of preference depended on the learned risk-reward environment and the payoff magnitude offered in the gambles (Fig. 3A). Specifically, participants in the negative condition chose the gamble more for low payoffs and less for high payoffs, in contrast to the uncorrelated condition $(b=1.99, \mathrm{CI}=[1.03,2.97]$, payoff $\times$ condition interaction $)$. 
These choices are consistent with participants in the negative condition inferring probabilities from payoffs, based on the risk-reward structure experienced. In the uncorrelated condition, participants tended to choose the sure thing irrespective of payoff magnitude $\left(M_{\text {sure }}=.19\right.$, gray line in Fig. 3A).

\subsubsection{Payoff-probability estimation task (test phase)}

Did inferred probabilities reflect previously learned risk-reward structures? Fig. 3B shows participants' estimates of the probability of winning a range of payoffs. A negative risk-reward relationship was observed in both conditions $\left(b_{\text {negative }}=-.78, \quad \mathrm{CI}=\right.$ $\left.[-.84,-.72], b_{\text {uncorrelated }}=-0.57, \mathrm{CI}=[-0.63,-0.51]\right)$, but it was stronger in the negative condition $(b=-0.22, \mathrm{CI}=[-0.30,-.13]$, condition $\times$ payoff interaction; in a regression with condition, payoff, and condition $\times$ interaction as predictors, using a normal link function). The results in the uncorrelated condition were unexpected in that the choice data suggest that participants had a strong prior expectation that they were not in an environment with a negative risk-reward structure.

To what degree did the learned relationship predict choices in the uncertainty task at the individual level? To investigate this, we first obtained a (risk-reward) slope for each participant through a random participant term when regressing probability estimates onto payoff magnitudes. This slope served as a measure of participants' judged risk-reward relationship. Steeper slopes indicate a stronger decrease in probability estimates as payoffs increase. This should lead to a stronger tendency to prefer the sure thing in the decisions under uncertainty task as the payoff magnitude increased. To examine this prediction, we ran a regression predicting choices from the risk-reward slopes, payoff, environmental condition, and the payoff $\times$ condition interaction. The regression showed that steeper risk-reward slopes predicted a stronger tendency to choose the sure thing as payoffs increased, but only for participants in the negative condition (payoff magnitude $\times$ slope, $b=2.58, \mathrm{CI}=[0.34,4.80])$. Individual risk-reward estimates in the uncorrelated condition were not associated with choosing the uncertain option (payoff magnitude $\times$ slope $\times$ uncorrelated interaction, $b=-2.09$, $\mathrm{CI}=[-4.79,0.62]$; modeled in a fixed effects logistic regression, results plotted in Supplement Fig. S13A). This result speaks against the possibility that participants in the uncorrelated condition used their subjective estimates across payoffs in decisions under uncertainty. Instead, they estimated an overall negative risk-reward relationship but were averse to uncertainty in their choices across payoffs.

\subsubsection{Recognition (test phase)}

Results from the decisions under uncertainty task imply that participants were somewhat sensitive to the negative risk-reward relationship. How did they learn that relationship? Did they memorize exemplars from the learning phase? Results from the gamble recognition task suggest that participants were overall unable to discriminate targets from foils. ${ }^{3}$ However, participants did show a bias toward stating that they recognized specific gambles (i.e., saying "Yes"): Of the eight gambles used to study the certainty effect, four fit the negative risk-reward structure (i.e., were "off" the slope) and four did not (see Fig. 2A). For gambles that were inconsistent with a negative risk-reward structure (i.e., structured as the bottom left and top right gambles in Fig. 2), participants tended to indicate not having seem them previously $\left(M_{\mathrm{yes}}=.28, b=-0.73, \mathrm{CI}=[-1.25,-0.22]\right)$. This effect was more pronounced for the negative condition $\left(M_{\text {yes }}=.17, b=-1.14\right.$, CI $=[-1.93,-0.37]$; logistic regression using risk-reward structure, condition, and their interaction as predictors, and participant as a grouping factor).

\footnotetext{
${ }^{3}$ Modeling the data in a signal detection theory framework makes this point clear: The discriminability parameter $d^{\prime}$ was centered at $0 \quad\left(M_{\text {negative }}=0.0\right.$, $\left.\mathrm{CI}=[-0.47,0.47], M_{\text {uncorrelated }}=0.00, b=0.00, \mathrm{CI}=[-0.67,0.67]\right)$. In addition, participants did not show any systematic response biases in either condition (criterion $\left.\left.c, M_{\text {negative }}=0.00, \mathrm{CI}=[-0.27,0.27]\right), M_{\text {uncorrelated }}=0.00, b=0.00, \mathrm{CI}=[-0.41,0.39]\right)$.
}

Thus, it is unlikely that participants encoded specific exemplars from the learning phase. Instead, participants in the negative condition may have abstracted a rule that they then used to assess the degree to which the stimuli were consistent with a negative risk-reward relationship. One limitation of the results from the gamble recognition task is that the stimuli set did not include any foils mimicking the structure of the certainty-effect gambles (namely, gambles located at the margins of the payoff-probability space). Instead, all of the extreme gambles were targets. We addressed this issue in the next experiment.

\subsection{Summary}

Experiment 1 exposed participants to either a negative or an uncorrelated risk-reward structure. The risk-reward structure led to environment-dependent preferences under uncertainty. In the negative risk-reward condition, participants were more likely to prefer the uncertain option with lower payoffs, and their learned risk-reward relationship explained this preference. In the uncorrelated condition, choosing the uncertain alternative was unrelated to payoff magnitudes and estimated risk-reward relationships. Finally, participants (incorrectly) reported not having seen gambles when those gambles were at odds with the negative risk-reward structure, suggesting that they had encoded the overall risk-reward structure as a rule, rather than encoding specific payoff-probability exemplars.

Surprisingly, a majority of participants in the uncorrelated condition estimated an overall negative risk-reward relationship in the estimation task. Though, we should emphasize, their estimates were less extreme than the negative condition and their choices in the decision making under uncertainty task did not reflect this pattern (both at the individual and group level). Nevertheless, we offer two possible explanations for the negative risk-reward relationship in the estimates for the uncorrelated condition. First, participants in the uncorrelated condition may have an ecologically informed bias to report a (negative) risk-reward relationship even when none exists (c.f., Langer, 1975). Second, although there was no risk-reward relationship across all gambles in the uncorrelated condition, there was what might be called a local risk-reward relationship within each trial of the learning phase. As participants chose between stochastically nondominated options in the learning phase, gamble A will always have a higher payoff but lower probability than gamble B, or vice versa. Thus, participants may have learned a risk-reward relationship from the local as opposed to the global risk-reward relationship. This was similar in the uncertainty task (the uncertain option was always larger than the sure thing). In Experiment 2, we modified the learning phase so that a local risk-reward relationship was not present.

\section{Experiment 2: Do people learn and exploit a positive risk-reward relationship?}

Experiment 2 sought to replicate and extend the finding that participants are sensitive to risk-reward relationships and harness them in making decisions under uncertainty. To do so, we added an environment with a positive risk-reward structure creating a rather blissful structure where the larger the payoff the more likely it is to occur. Given such an idealistic structure is arguably less prevalent outside the lab, it can provide a stronger test of how well participants adapt to different risk-reward structures. The additional positive risk-reward environment affords a stronger test of how risk-reward environments may create environment-dependent preferences. Specifically, participants in the negative condition should prefer the uncertain option for low payoffs and the sure thing for high payoffs. Conversely, participants in the positive condition should prefer the sure thing for low payoffs and the uncertain option for high payoffs. Additionally, preferences for the uncertain option in the uncorrelated condition would be independent of payoff magnitudes.

To create a learning phase without a local risk-reward structure, we 

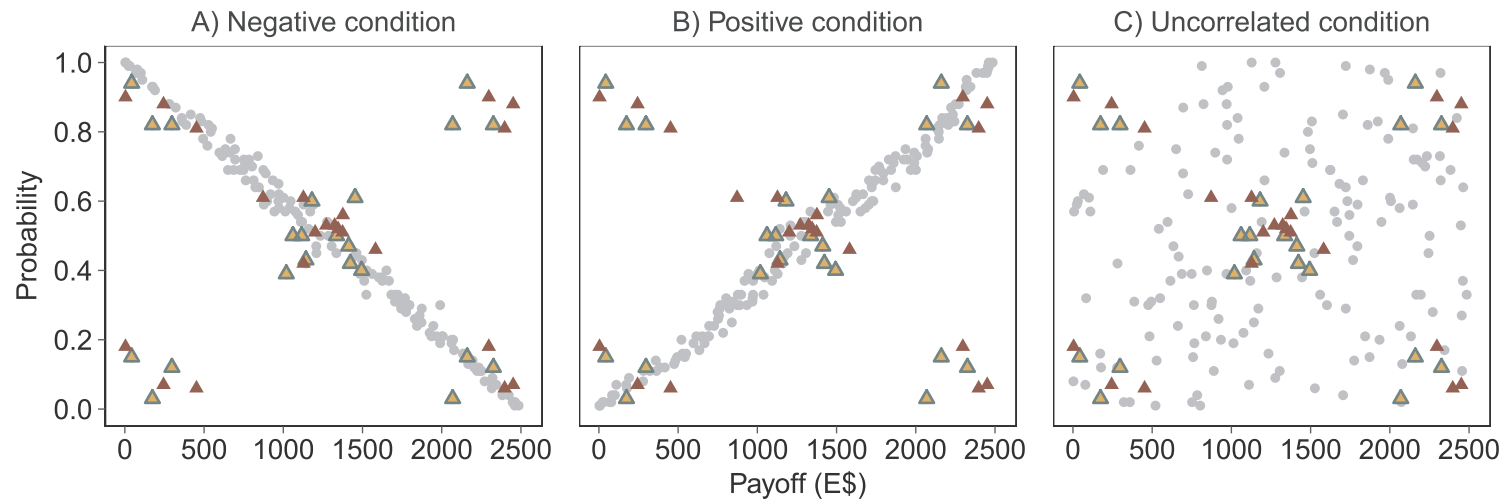

- Learning environment $\bullet$ Common $\Delta$ Recognition target $\Delta$ Recognition foil

Fig. 4. Stimuli used in Experiment 2. The learning phase consisted of 150 condition-dependent gambles, and 22 common gambles (triangles).

asked participants-instead of choosing between two gambles as in Experiment 1-to state the price for which they would be willing to sell (WTS) a single gamble presented at each trial for. Based on our findings from Experiment 1, we predicted that participants would learn about risk-reward relationships incidentally while pricing the gambles.

Finally, we sought to better understand the degree to which participants encoded the risk-reward relationship as a rule. We did so by modifying the gamble recognition task so that target and foil gambles were structured equally, especially at the four extremes (Fig. 4). We hypothesized that participants would not distinguish between targets and foils-which would be difficult to do-but would respond based on the gambles' fit with previously experienced risk-reward structures.

\subsection{Method}

\subsubsection{Participants}

We recruited 90 participants (53 females, mean age 24.7, $S D=4.1$, proportion students $=.72$ ) from the participant pool at the Max Planck Institute for Human Development. Each participant completed the experiment in exchange for a show-up fee of $10 €$ and a performancecontingent bonus. Participants in Experiment 1 were excluded from the recruitment process.

\subsubsection{Decisions under risk (learning phase)}

The methods were largely the same as in Experiment 1; here, we summarize key differences. We used a larger payoff range (E $\$ 1.01-2500$, disclosed conversion rate $\mathrm{E} \$ 2500=1 €)$. Fig. 4 depicts the three risk-reward environments used in the current experiment (150 gambles per environment with identical marginal distributions; see Supplementary Material for details, and code on the OSF). Briefly, to create the positive risk-reward condition, we took the gambles in the negative condition and reversed the order of probabilities such that the highest probabilities were now associated with the highest payoffs and vice versa.

To study condition-dependent differences in how participants priced (identical) gambles, we included 22 gambles common to all three conditions (10 in the center, 3 at each margin; see triangles in Fig. 4). In total, this procedure resulted in 172 risky gambles per risk-reward condition while controlling for the marginal distribution of payoffs and probabilities across all three conditions.

\subsubsection{Decisions under uncertainty (test phase)}

For the uncertainty task, we created gamble pairs with low (E\$1-250), intermediate (E\$1125-1375), and high payoffs (E\$2250-2500) (10 pairs each). As in Experiment 1, the uncertain option's payoff (probability "?") was half as big as the certain option's payoff. In a typical pair, participants chose between a $100 \%$ chance of winning $\mathrm{E}$ $\$ 50$ and a “?" chance of winning $\mathrm{E} \$ 100$. We included 30 filler trials in which the certain option was created by scaling down the uncertain option by a random factor between .1 and.9.

\subsubsection{Payoff-probability estimation task (test phase)}

We increased the number of trials such that participants estimated the probabilities associated with 20 payoff magnitudes (range E \$1-2500). To investigate how well participants learned the bi-directional relationship between payoffs and probabilities, we also asked participants to estimate the payoff associated with a given probability at the end of the experiment (probability-payoff estimation task). We drew 20 probabilities between 0 and 1 for this task (results mirrored the results of the probability estimation task, and are reported in the Supplementary Material).

\subsubsection{Recognition (test phase)}

We used the gambles common to both conditions in the learning phase as targets and an equally generated set of gambles as foils (Fig. 4, red triangles). Thus, foils were 10 gambles at the center and 12 gambles at the margins of the payoff-probability distribution space (novel random draws based on the recognition gambles procedure). This broader set (relative to Experiment 1) of 22 targets and 22 equally structured foils was used to test whether the risk-reward relationship was learned via exemplars or a rule: If participants had learned the relationship as a rule, they should not indicate having seen gambles that did not fit condition-dependent risk-reward structure (and indicate having seen gambles that did) irrespective of whether those gambles were targets or foils.

\subsubsection{Procedure}

During the learning phase, participants indicated their WTS for one gamble at a time. They took self-paced breaks after each of five blocks. Common gambles were randomly interspersed after 100 condition-dependent trials. The task was presented as a game show called "Keep or Sell?" ("Behalten oder Verkaufen?"). To motivate participants to indicate their true valuations of a gamble, we implemented a BeckerDeGroot-Marschak auction (Becker, DeGroot, \& Marschak, 1964). The rules were as follows. Participants owned the right to play each gamble, which they could sell to the experimenter at a price they determined themselves. Prices were entered with a mouse click on a rating scale (E $\$ 0-2500$ ) and confirmed with a click on the value.

To incentivize the task, we informed participants that 10 gambles would be randomly selected and played out at the end of the experiment. The experimenter then offered a (computer-generated) buying price between 0 and the maximum payoff from the gamble. If the experimenter's price exceeded the participant's selling price, the participant sold the gamble and earned the buying price. If the participant's selling price exceeded the experimenter's buying price, the gamble was played out (e.g., $50 \%$ chance of $\mathrm{E} \$ 380$ ). The dominant strategy in this 

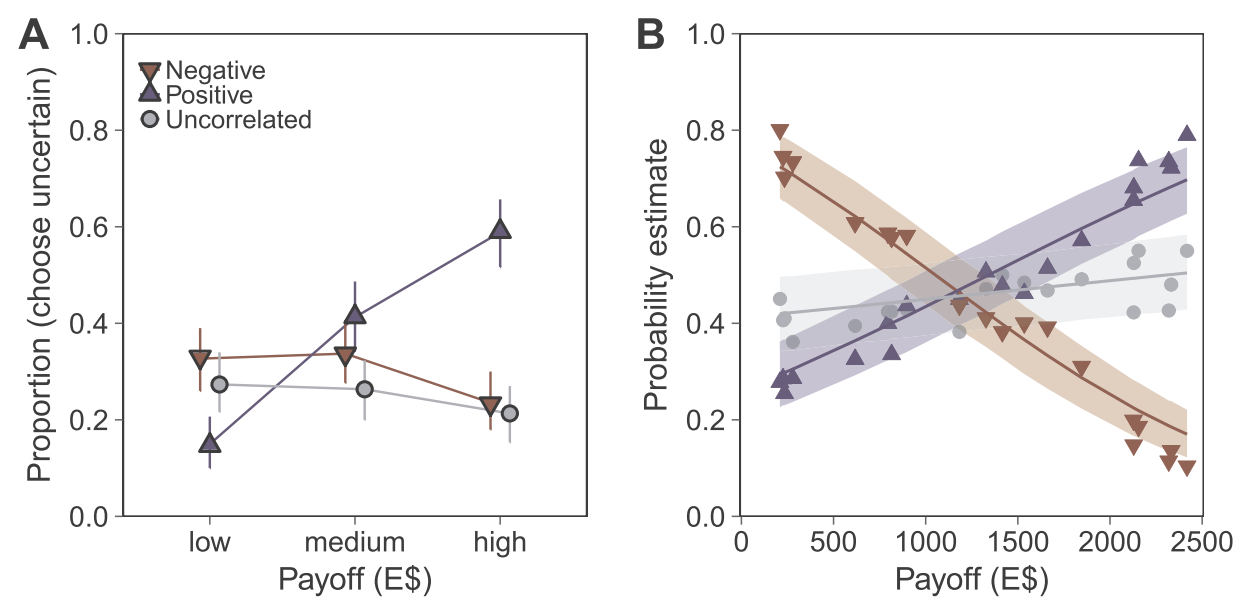

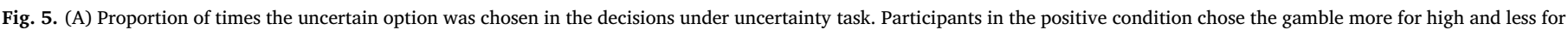

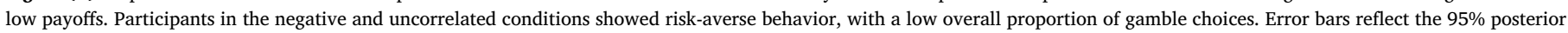

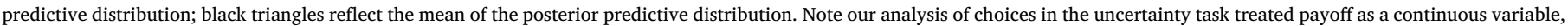

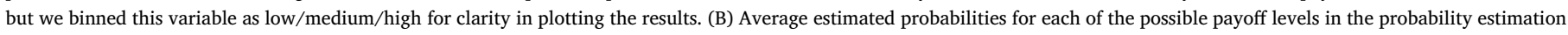

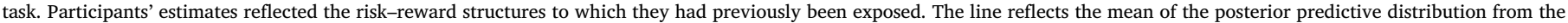
linear regression; ribbons reflect the $95 \%$ posterior predictive distribution.

task is to price a gamble based on its subjective value: Higher prices can prevent participants from selling unattractive gambles; lower prices can lead to them selling attractive gambles under value. In other words, the prices should approximate participants' subjective certainty equivalents for the gambles.

Participants completed five practice trials to ensure their proper understanding of the WTS measure. If they indicated a selling price that exceeded the maximum payoff from that gamble, participants would see a screen reminding them that (i) they would only receive counteroffers between 0 and the maximum amount to be gained in the gamble, (ii) setting an accurate price would increase the likelihood of good counteroffers, and (iii) good counteroffers would maximize the bonus to be gained from the task. After this feedback, participants set a new price for the same gamble. If they had no more questions, they proceeded to the main part of the task, in which there was no feedback.

The test phase in Experiment 2 was equivalent to that in Experiment 1 (see Table 1 for an overview of the tasks and task order). The exception was that the decisions under uncertainty task was now incentivized. In particular, five choices from the uncertainty task were randomly selected and played out. If the uncertain option was chosen, then the condition-dependent probabilities were used to determine the probability of the outcome. Participants were instructed about the incentivization scheme at the beginning of the task. At the end of the experiment, we played out the randomly drawn trials from the learning phase and the uncertainty task. Bonuses (between $1.99 €$ and $7.82 €$, with $\mathrm{E} \$ 2500=€ 1$ ) were added to the regular payment.

\subsection{Results}

\subsubsection{Decisions under risk (learning phase)}

Across all gambles, including the environment gambles, prices were strongly related to the gambles' expected values (indicated by a credible payoff $\times$ probability interaction, $b=0.88, \mathrm{CI}=[0.85,0.91])$. Prices in the positive condition deviated slightly more from expected values compared to in the other two conditions (payoff $\times$ probability $\times$ positive condition, $b=-.07, \mathrm{CI}=[-.12,-.03])$. However, these differences did not persist when we modeled certainty equivalents given for the subset of gambles common to all conditions (thereby controlling for condition-dependent stimuli features). In addition, there were no differences in participants' subjective evaluations of payoffs and probabilities as modeled by prospect theory (Tversky \& Kahneman, 1992) (see Supplementary Material). In sum, and consistent with Experiment 1, participants seemed to evaluate risky gambles in a similar manner across conditions.

\subsubsection{Decisions under uncertainty (test phase)}

How did the experienced risk-reward structures shape participants' preferences under uncertainty? Fig. 5A displays the proportion of choices of the uncertain option as a function of the possible payoff level, separately for the three conditions. In general, participants were more likely to choose the certain but smaller payoff option over the uncertain option that offered a larger payoff $\left(M_{\text {sure }}=.63, b=1.97\right.$, CI $=$ $[1.43,2.55])$. However, this preference depended on the risk-reward environment to which participants had previously been exposed and on the payoff magnitude offered in the gambles. Consistent with our prediction of environment-dependent preferences, the higher the payoffs, the more often participants in the positive condition chose the gamble $\left(b_{\text {positive }}=3.04, \mathrm{CI}=[2.41,3.69]\right.$, condition $\times$ payoff interaction $)$. When payoffs were high, participants in the positive condition chose the uncertain option in as many as $59 \%$ of trials.

As Fig. 5A shows, the pattern of results was very different for participants in the negative condition, who chose the gamble slightly more for smaller payoffs and less for larger payoffs. Nevertheless, unlike the results of Experiment 1, the effects in the negative condition were rather small, and the choices were not credibly different from those in the uncorrelated condition $\left(b_{\text {negative }}=-.22, \mathrm{CI}=[-.84, .41]\right.$; all effects modeled in a logistic regression with the uncorrelated condition as baseline). This finding was unexpected: If participants had relied on the learning phase and exclusively used the knowledge they expressed in their probability estimates in the choice task, they should have been much more risk seeking for low payoffs, which they would have learned to be associated with high probabilities. As we will show shortly, this may be due to (or linked back to) individual variability in the learned risk-reward relationship. ${ }^{4}$

\subsubsection{Payoff-probability estimation task (test phase)}

Did participants' estimates reflect risk-reward environments from the learning phase? As Fig. 5B shows, the probabilities that participants estimated varied as a function of the possible payoffs. Participants in the uncorrelated condition estimated a weak positive relationship $(b=0.11, \mathrm{CI}=[0.06,0.16])$. Estimates from participants in the other two conditions reflected the specific risk-reward structure to which

\footnotetext{
${ }^{4}$ The choice patterns for the filler gambles were identical to these results (positive condition choosing gambles more as payoffs increase; negative condition similar to uncorrelated condition); as expected, choices here also largely depended on the difference between certain and uncertain payoffs $\left(b_{\text {uncertain }} /\right.$ certain $\left.=-8.84, \mathrm{CI}=[-9.56,-8.16]\right)$.
} 


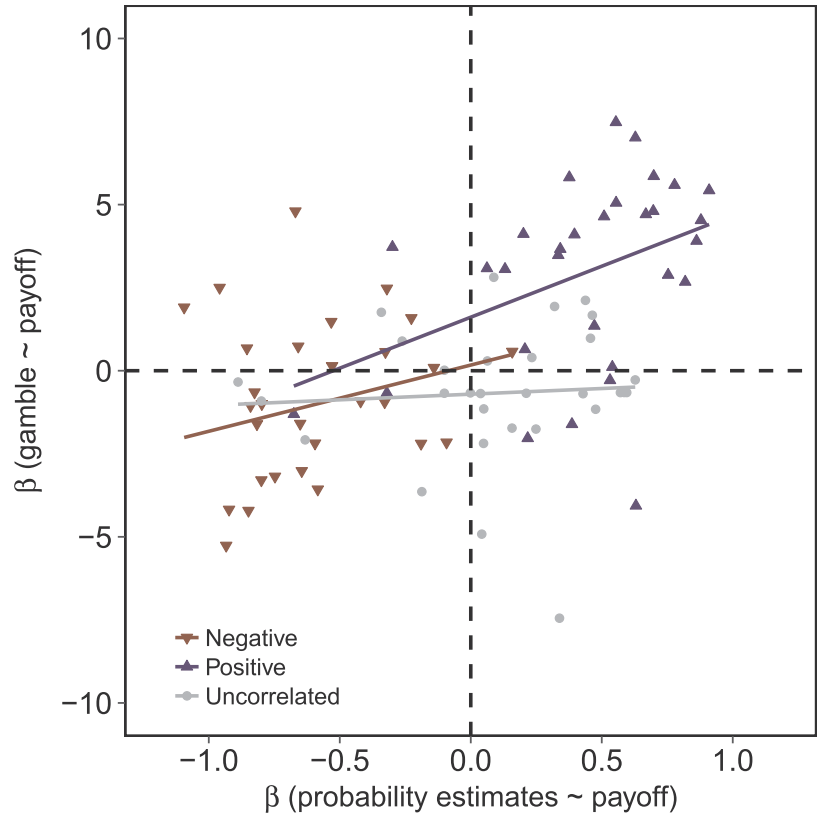

Fig. 6. Individual variation in choice of the uncertain alternative (y-axis) based on estimated payoff-probability relationships (x-axis). Each data point depicts one participant. The participant-level $\beta$ was estimated from a Bayesian regression with a random participant intercept for (estimates $\sim$ payoff) and (choice $\sim$ payoff), respectively. Values above 0 indicate higher probability estimates with increasing payoffs (x-axis), and a preference for the uncertain option with increasing payoffs (y-axis), and vice versa values below 0 . Payoff-probability estimates in the negative and positive condition, but not in the uncorrelated condition, predicted choice.

these participants had previously been exposed. In the negative condition, the estimates showed a negative relationship between payoffs and probabilities $(b=-0.75, \mathrm{CI}=[-0.82,-0.68])$. In the positive condition, the reverse applied but the slope was much shallower $(b=0.35$, $\mathrm{CI}=[0.28,0.42]$, in a normal link regression using condition, payoff, and condition $\times$ payoff as predictors). The shallower slope was partially driven by two participants in the positive condition who estimated a negative risk-reward relationship, perhaps indicative of a possible negative risk-reward relationship 'default'/prior.

As in Experiment 1, we also investigated the extent to which an individual's probability estimates predicted his or her choices in decisions under uncertainty. To do so, we again obtained a (risk-reward) slope for each participant through a random participant term when regressing probability estimates onto payoff magnitudes. As Fig. 6 shows, the majority of slopes (plotted on the x-axis) reflected the condition to which participants had been exposed: the negative condition's slopes fell in the negative range; the positive condition's slopes fell in the positive range. We then used the individual slopes to predict choosing the uncertain over the certain option across different payoff magnitudes. The risk-reward slopes predicted payoff-dependent preferences for the uncertain option in the two correlated conditions $\left(b_{\text {negative }}=2.01, \mathrm{CI}=[0.27,3.76] ; b_{\text {positive }}=2.74, \mathrm{CI}=[1.03,4.45]\right.$, slope $\times$ payoff $\times$ condition interaction in a fixed effects model using the uncorrelated condition as baseline). That is, following up on the degree to which the risk-reward conditions impacted preferences, once individual variability in the learned risk-reward relationship is accounted for, preferences in both the negative and positive condition were credibly different (and in the predicted direction) from the uncorrelated condition. Nevertheless, the link between estimates and choices was weaker in the negative condition (red vs. blue slope in Fig. 6). As in Experiment 1, risk-reward slopes in the uncorrelated condition did not predict the choice of an uncertain option $\left(b_{\text {uncorrelated }}=.44\right.$, CI $=$ $[-.56,1.43])$.

\subsubsection{Recognition (test phase)}

Results from the decisions under uncertainty and estimation tasks suggested that participants learned risk-reward structures in the first phase of the experiment. But how did they represent the different structures? A comparison of panels A and B in Fig. 7 shows that participants responded similarly when the gambles presented were targets versus foils, implying that they could not discriminate between them. ${ }^{5}$

When we broke responses down by whether or not gambles fit a condition's risk-reward structure (Fig. 7), the results resembled those of Experiment 1: If gambles did not fit a condition-dependent risk-reward structure, participants indicated that they had not seen them previously, irrespective of whether these gambles were targets or foils. That is, a majority of participants in the negative condition reported not having seen gambles that were consistent with a positive risk-reward relationship $\left(M_{\mathrm{yes}}=.26, b=-1.40, \mathrm{CI}=[-1.80,-1.00]\right)$. Conversely, a majority of participants in the positive condition reported not having seen gambles that were consistent with a negative risk-reward relationship $\left(M_{\mathrm{yes}}=.26, b=-.85\right.$, CI $\left.=[-1.25,-.46]\right)$.

What is more, participants were also more likely to report having seen gambles merely because their structure followed a condition's risk-reward structure, again irrespective of whether these gambles were targets or foils (see Fig. 7). That is, participants in the negative condition were likely to report having seen a gamble if the gamble was consistent with a negative risk-reward relationship $\left(M_{\text {yes }}=.65, b=.84\right.$, $\mathrm{CI}=[.45,1.23])$, and participants in the positive condition were likely to report having seen a gamble if the gamble was consistent with a positive risk-reward relationship $\left(M_{\mathrm{yes}}=.66, b=.52, \mathrm{CI}=[.13, .90]\right.$; all results from a logistic regression using condition $\times$ stimulus type as a predictor, neutral gambles in the uncorrelated condition as baseline). Responses to neutral gambles were identical across conditions. These gambles were consistent with all risk-reward structures, which might explain why people were equally likely to indicate that they had previously seen them (in all three conditions; $M_{\mathrm{yes}}=.68$, bars on the right in Fig. 7A and B).

\subsection{Summary}

Experiment 2 substantiated the findings from Experiment 1 that participants could learn risk-reward structures in an unsupervised, incidental fashion, and that they subsequently often exploited the relationship to make decisions under uncertainty. In particular, we showed that participants learned and used a positive risk-reward relationship, although this structure stands in stark contrast to the negative risk-reward relationship present in many real-world environments. Moreover, in contrast to Experiment 1, where probability estimates in the uncorrelated condition showed a negative association with payoff levels, probability estimates were now independent of payoff levels. Comparing Experiment 2 to Experiment 1 suggests that this difference is due to the learning phase, in which participants now evaluated one gamble at a time, removing any 'local' risk-reward structure naturally built into a choice task with nondominated gambles. In a payoff-probability estimation task participants used risk-reward relationships to infer probabilities from payoffs-and that the risk-reward relationship learned from pricing gambles dictated the direction of the estimates. Finally, we found further evidence of environmentdependent preferences in decisions under uncertainty. One qualification to this result is that participants in the negative condition were not as keen on choosing the uncertain option for low payoffs as we had expected (despite estimating high probabilities for these payoffs).

\footnotetext{
${ }^{5}$ A signal detection analysis showed that participants did not discriminate between old and new gambles across all three conditions ( $d_{\text {uncorrelated }}^{\prime}=0.21, \mathrm{CI}=[-0.17,0.60]$; $d_{\text {positive }}^{\prime}=-0.16, b=-0.37, \quad \mathrm{CI}=[-0.89,0.15] ; \quad d_{\text {negative }}^{\prime}=-0.05, b=-0.26$, $\mathrm{CI}=[-0.78,0.27])$. There were weak, but not credible, biases towards saying 'yes' in the correlated conditions ( $\left.c_{\text {uncorrelated }}=-0.10, \mathrm{CI}=[-0.30,0.08]\right), c_{\text {positive }}=0.08, b=0.18$, $\left.\mathrm{CI}=[-0.09,0.45], c_{\text {negative }}=0.03, b=0.13, \mathrm{CI}=[-0.14,0.40]\right)$.
} 
A

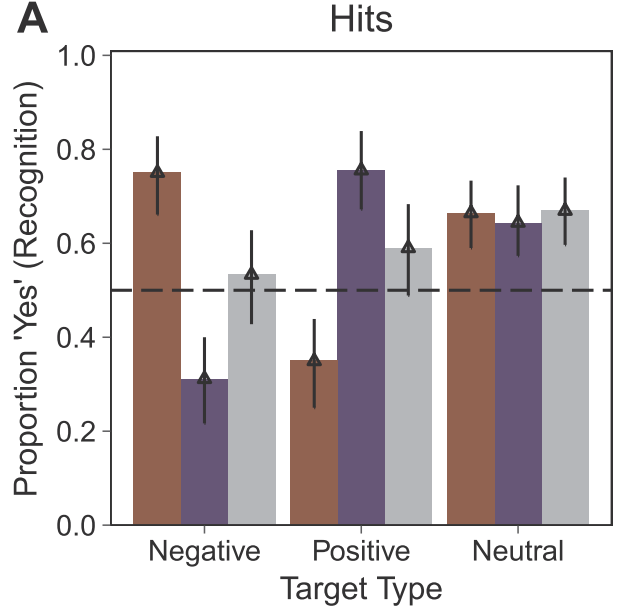

B

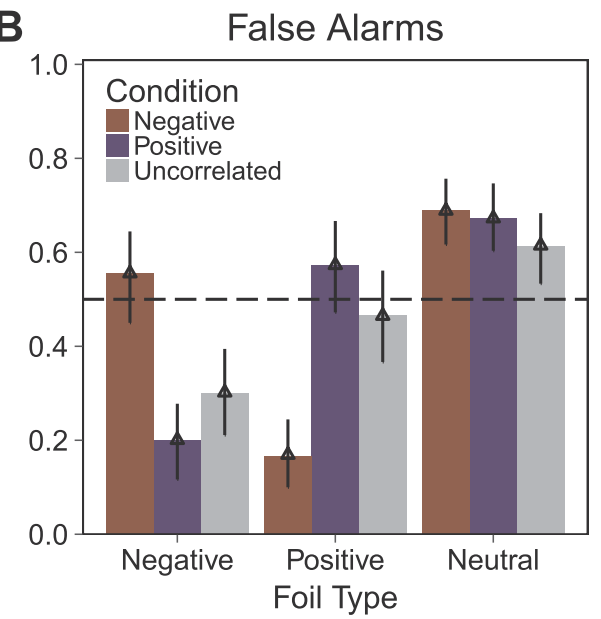

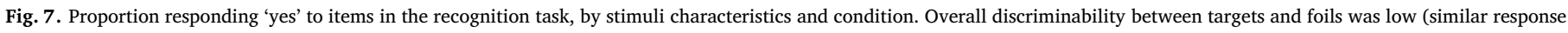

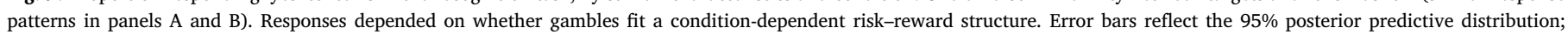
triangles reflect the mean of the posterior predictive distribution.

Finally, the recognition task in Experiment 2 provided further evidence that the risk-reward relationship from the learning phase was represented as a rule rather than in terms of memorized gambles.

Across Experiments 1 and 2, the gambles used in the learning phase presented risks in terms of explicit, single numbers. Outside the laboratory, in contrast, many gambles are about epistemic events, for instance when betting on the outcome of a sporting event (e.g., a soccer match). To gauge their chances of winning for such gambles, people may rely on the prior knowledge they have about these events. In our final study, we asked how well our results generalized to choices about these epistemic events.

\section{Experiment 3: Do people learn about and exploit risk-reward structures from gambles about epistemic events?}

In Experiment 3, we examined whether people learn risk-reward relationships when the chances of winning depend on events about which they have some prior knowledge. Specifically, in both the learning and test phase, we used gambles in which winning was tied to the maximum temperature in Berlin on a particular day in 2011 falling within a certain range (e.g., "You win $\mathrm{E} \$ 500$ if the temperature on August 20th, 2011, was between 16 and $\left.25^{\circ} C^{\prime \prime}\right)$. We adapted a procedure from Tversky and Fox (Tversky \& Fox, 1995) to create different events using different widths and locations of temperature ranges, so that participants should a priori have different subjective probabilities of the events occurring (see also Tversky \& Wakker, 1995). As in Experiment 2, participants were asked to state prices for gambles in the learning phase. To create different risk-reward relationships, we determined the probability that a given interval would contain the maximum temperature based on the width of the interval and its proximity to the mean August temperature. We refer to these probabilities as historical frequencies. We paired them with payoffs between $\mathrm{E} \$ 1.01$ and $\mathrm{E} \$ 2500$ to create either a positive or a negative risk-reward relationship. Using these two conditions, we aimed to extend our finding that participants learn risk-reward relationships incidentally from simple monetary gambles to gambles with epistemic events.

Learning about risk-reward relationships from implied subjective beliefs alone may be challenging. Moreover, in some situations, probability estimates about epistemic events are available, such as when an meteorologist shares her belief that an event will occur. Thus, we further differentiated the learning environments, with half the participants being shown only the temperature range of the event but no explicit probability information ('learning under uncertainty') and half additionally being shown the historical frequencies ('learning under risk').
By comparing these two sets of conditions, we tested whether explicit probability information is necessary to learn the risk-reward relationship.

In the test phase, we assessed the influence of the risk-reward environments somewhat differently compared to the first two experiments. Because the chances of the maximum temperature in Berlin falling within a particular temperature range could be inferred from the range itself, we tested for the effect of the risk-reward environment on probability estimates and choices above and beyond the information provided by the temperature range (historical frequencies). How can environment-dependent preferences emerge here? In the negative risk-reward environment, the proportion of choices of the uncertain option should increase for lower payoffs, and decrease for higher payoffs-and vice versa for the positive risk-reward environment. A similar pattern should emerge for probability estimates: When relying on a negative risk-reward structure, probability estimates for an event associated with a low payoff should be larger than probability estimates for an (otherwise comparable) event associated with a high payoff. When relying on a positive risk-reward structure, the opposite should happen.

Lastly, we examined whether participants generalized learned risk-reward relationships to other contexts. To this end, we added tasks in which participants made decisions under uncertainty and estimated probabilities about the maximum temperature falling in a particular range in Dushanbe, Tajikistan. Importantly, our results showed that participants did not rely on risk-reward structures in a context they had not been exposed to (for details see the Supplementary Material).

\subsection{Method}

\subsubsection{Participants}

We recruited 200 participants from the participant pool at the Max Planck Institute for Human Development in Berlin (125 females, mean age $=24.45, S D=4.3$, proportion students $=.84$ ) to take part in the experiment for a $10 €$ show-up fee and a performance-contingent bonus. Participants in Experiment 1 and 2 were excluded from the recruitment process. Due to the change in design, we expected smaller effect sizes and therefore increased our sample from 30 to 50 participants per condition. Participants were randomly assigned to one of four conditions. Due to a computer error, the responses from two participants in the uncertainty task were not saved (leaving $N=198$ ).

\subsubsection{Decisions under uncertainty $v$ s. risk (learning phase)}

During the learning phase, participants priced gambles based on the 
Berlin weather. To construct these gambles, we retrieved past weather data on the mean $\left(M=22.7^{\circ} \mathrm{C}\right)$ and standard deviation $\left(S D=3.2^{\circ} \mathrm{C}\right)$ of the maximum daily temperature in August in Berlin in 2011 from $a c$ cuweather.com. We created 155 temperature ranges of varying width and location on the temperature scale (see Supplementary Fig. S6). Because the maximum temperatures were approximately normally distributed, we calculated the historical frequency to approximate the probability that the maximum temperature on a given date would fall within the specified interval. We then constructed gambles such that there was either a positive or a negative risk-reward relationship holding the marginal distributions of payoffs and historical frequencies constant across conditions (see Supplementary Material S7, code on OSF).

In the learning under uncertainty condition, only the temperture range and the corresponding payoff was shown for each gamble (e.g., "E\$2300 if the maximum temperature was between 13 and $15^{\circ} \mathrm{C}$ on Aug 29th".) In the learning under risk condition, the historical (relative) frequency was added to the gamble (e.g., "E\$2300 if the maximum temperature was between 13 and $15^{\circ} \mathrm{C}$ on Aug 29th ( $\left.p=3 \%\right)$."). Screenshots are shown in Supplementary Material S8.

\subsubsection{Decisions under uncertainty (test phase)}

We created a decisions under uncertainty task in which participants chose between an uncertain option that depended on the Berlin weather event having occurred ("E\$2000 if the maximum temperature was between 23 and $26^{\circ} \mathrm{C}$ on August 22nd") and a smaller, sure thing ("700 E\$ for sure"). We varied the payoffs on two levels, to be either high (E $\$ 2000$ vs. $\mathrm{E} \$ 700$ for sure) or low (E\$100 vs. $\mathrm{E} \$ 35$ for sure). Participants completed 15 different trials about Berlin weather.

\subsubsection{Subjective probability estimation task (test phase)}

This task consisted of two parts. First, participants were asked to estimate their subjective probability $(0-100 \%)$ of winning the gamble (i.e., the event occurring) in the decisions under uncertainty task with payoff information. Our key interest was the degree to which participants used the payoff information in their estimates. Participants were therefore shown the actual gamble (e.g., "E\$2000 if the maximum temperature in Berlin was between 23 and $26{ }^{\circ} \mathrm{C}$ on August 22nd") and asked to judge the probability that they would win.

In a second part, participants indicated their subjective probability $(0-100 \%)$ that the maximum temperature on a given day in August would fall in a given temperature range without payoff information (e.g., "likelihood the maximum temperature in Berlin was between 23 and $26^{\circ} \mathrm{C}$ on August 22nd"). The temperature ranges were identical to those used in the decisions under uncertainty task and in the subjective probability estimation task with payoff information.

\subsubsection{Payoff-probability estimation task (test phase)}

Participants were presented with 20 different payoffs and asked to think back to the gambles they had experienced in the learning phase. They then estimated the likelihood of winning these payoffs in the upcoming bonus trials. This task was used to test whether participants had picked up on the risk-reward structures in the learning phase.

\subsubsection{Procedure}

Participants were randomly assigned to one of four learning conditions (Negative Risk, Negative Uncertainty, Positive Risk, Positive Uncertainty). They evaluated gambles about the maximum temperature measured on a given day in August 2011 by indicating a WTS for each gamble. The instructions were adapted from Experiment 2. Here, participants were informed that a gamble's value was determined by the extent to which the temperatures were in line with the true temperatures on a given day, and by its possible payoff. The instructions for the risk conditions included an explanation of the historical frequency information added to the gambles, namely that the probability was based on typical August temperatures (i.e., " $45 \%$ is the likelihood that a typical August day will fall in the temperature range given in the bet"). During the learning phase, participants took self-paced breaks between five blocks of 31 pricing trials each. The learning phase was incentivized such that prices from 10 randomly drawn trials were played out according to the Becker-DeGroot-Marschak auction procedure described in Experiment 2, but now the outcome of the gamble was determined by whether the event's temperature range actually contained the true maximum temperature.

The order of tasks in the test phase was identical across all participants. We randomized the positions of sure things versus gambles in the decisions under uncertainty task, as well as the position of the payoff amount in the gamble (above or below the event) on the trial level. The decisions under uncertainty task was incentivized such that five randomly selected choices were played out. At the end of the experiment, bonuses (between $1.28 €$ and $7.65 €$, with $\mathrm{E} \$ 2500=€ 1$ ) were added to the regular payment.

\subsection{Results}

\subsubsection{Decisions under risk vs. uncertainty (learning phase)}

The prices suggested that participants traded off the payoff and the historical frequencies of events (effect of EV, defined as historical frequency $\times$ payoff: $b=.46, \mathrm{CI}=[.40, .53])$. As expected, risky gambles that included information about historical frequencies (negative risk and positive risk conditions) were closer to the EVs of the bets than their uncertain counterparts $\left(b_{\text {negative uncertain }}=-.66\right.$, $\mathrm{CI}=[-.76,-.57], b_{\text {positive uncertain }}=.58, \mathrm{CI}=[.48, .67] ; 3$-way interaction using a gamble's $\mathrm{EV} \times$ risk-reward relationship $\times$ type of learning).

\subsubsection{Decisions under uncertainty (test phase)}

Did the experienced risk-reward relationships shape preferences under uncertainty? We expected this to be the case after "learning under risk" (as in Experiments 1 \& 2), in particular, but also (though less strongly) after learning under uncertainty. We analyzed conditiondependent choices after controlling for the events' historical frequencies. ${ }^{6}$ Fig. 8 shows the results of this analysis. Overall, participants were less likely to choose the gamble over the sure outcome for high (E $\$ 2000)$ than for low payoffs ( $\mathrm{E} \$ 100)$ across conditions $\left(b_{\mathrm{E} \$ 2000}=-1.23\right.$, $\mathrm{CI}=[-1.62,-.85])$. Was this payoff effect moderated by learned risk-reward structures? Indeed, consistent with the risk-reward relationship they had experienced in the learning phase, this payoff effect was smaller for participants who had been exposed to a positive risk-reward relationship under risk (panel A). This effect was driven by participants in the positive condition choosing the gamble less often when the choice was associated with a E $\$ 100$ payoff-a payoff that had previously been associated with a low probability $\left(M_{\text {gamble }}=-.12, b_{\text {positive }} \times \mathrm{E} \$ 100=-.57, \mathrm{CI}=[-1.08,-.05]\right.$, all results based on a mixed effects logistic regression controlling for historical frequencies, using learning type [risk vs. uncertain] $\times$ risk-reward relationship [negative vs. positive] $\times$ payoff level as predictors).

Learning under uncertainty did not affect choice. In sum, there is some evidence for environment-dependent preferences, namely when participants were exposed to the risk-reward relationship under risk. Participants in the positive condition became less risk seeking for low payoffs but not more risk seeking for high payoffs, as one would have expected from Experiments 1 and 2.

\subsubsection{Subjective probability estimation tasks (test phase)}

Participants were also asked to estimate the chances that a maximum temperature would fall within a given temperature range both within the context of the gamble as a whole (including payoff information associated

\footnotetext{
${ }^{6}$ Choices were well-adjusted to the events' historical frequencies, with an almost linear increase in the proportion of participants choosing the uncertain option as probabilities of winning based on historical frequencies increased $\left(b_{\text {probability }}=6.98, \mathrm{CI}=[6.48,7.51]\right.$, see Supplementary Material S11 for posterior predictions across different historical frequencies).
} 


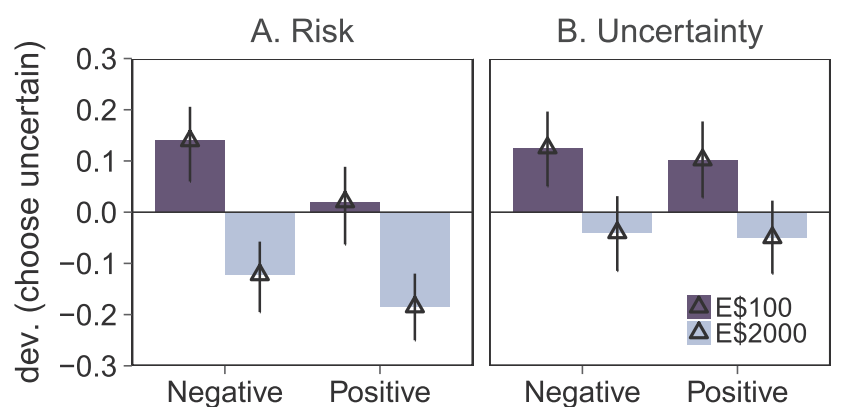

Fig. 8. Decisions under uncertainty. Plots show how much participants picked the uncertain gamble after controlling for the gamble's historical frequencies. Choice proportions perfectly adjusted to the gambles' historical frequency should have a 0 deviation. Bars and triangles reflect the mean of the posterior predictive choice distribution (controlling for historical frequency); error bars indicate the $95 \%$ posterior predictive distribution (generated using historical frequencies of .5).
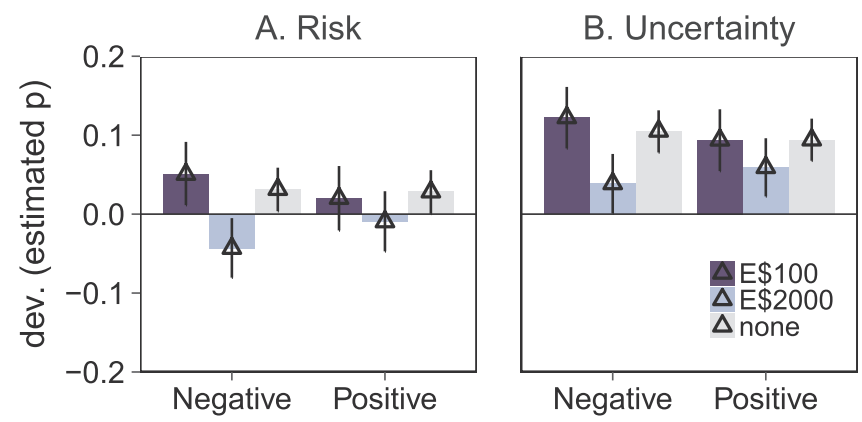

Fig. 9. Subjective probability estimation tasks. Plots show deviations of participants' estimates after controlling for the gambles' historical frequencies. Estimates perfectly in line with the gambles' historical frequency should have a deviation of 0 . Participants gave subjective probability estimates of winning a particular temperature bet including a payoff (blue bars) or the probability of a given temperature range alone (gray bars). Bars show posterior mean deviations from historical frequencies; error bars show $95 \%$ highest density intervals (generated using historical frequencies of .5).

with the event) and without this payoff information. As we were interested in how the estimates were affected by the risk-reward environments after controlling for the historical frequencies associated with the events, we report deviations from those historical frequencies. ${ }^{7}$ Did participants rely on previously experienced risk-reward structures when gauging their chances of winning a bet about the weather? Fig. 9 (A, B) shows that participants' subjective estimates were indeed guided by the payoff information. In line with our predictions and Experiments 1 and 2, in the negative conditions (panel A, left bars), subjective probability estimates were lower when temperature ranges were presented in a gamble context that offered a $\mathrm{E} \$ 2000$ payoff $(b=-.10, \mathrm{CI}=[-.12,-.07])$ than in a gamble context that offered a $\mathrm{E} \$ 100$ payoff.

This payoff effect-a difference in estimates for $\mathrm{E} \$ 2000$ vs. $\mathrm{E} \$ 100$ after learning under risk-was not observed in the positive condition (panel A, right bars, $b=-.04, \mathrm{CI}=[-.09, .03]$ modeled in a normal link regression using learning type [risk vs. uncertain] $\times$ risk-reward relationship [negative vs. positive] $\times$ payoff level as predictors). As Fig. 9 further shows-and contrary to our predictions-the payoff effect did not flip (with higher payoffs leading to a positive deviation and lower payoffs leading to a negative deviation). A bi-product of this was that participants in the risky positive condition ended up with estimates closer to the true historical frequencies (Fig. 9, panel A). For participants who had learned about risk-reward relationships under uncertainty (panel B), the between-condition effects were comparable

\footnotetext{
${ }^{7}$ All participants were sensitive to historical frequencies and provided estimates that reflected these frequencies across contexts $(b=.77, \mathrm{CI}=[.76, .79]$, see Supplementary Material S12).
}

(larger payoff effect in the negative condition, see panel B).

Did probability estimates in this task predict choices in the decisions under uncertainty task? Indeed, we found a positive link between estimates and choices $(b=4.00, \mathrm{CI}=[3.25,4.78]$, main effect of estimate in a logistic regression using historical frequencies, estimates, and their interaction as predictors).

\subsubsection{Payoff-probability estimation task (test phase)}

To what extent did probability estimates reflect the experienced risk-reward structures? Fig. 10 shows that, as expected, the estimates of participants in the negative condition reflected the risk-reward structure from the learning phase (slope in the negative condition = $b_{\text {negative }} \times$ payoff $\left.=-.40, \mathrm{CI}=[-.45,-.35]\right)$. Estimates in the positive conditions were regressive to $50 \%$ (slope $=.02, b_{\text {positive }} \times$ payoff $=.42$, $\mathrm{CI}=[.35, .49]$, interaction effect using the negative condition as baseline). Fig. 10 (panels A vs. B) also shows that the results were identical for the risky and uncertain learning conditions $\left(b_{\text {uncertain }}=.02\right.$, CI $=$ $[-.03, .08]$, all estimates modeled in a normal link regression, using learning type (risk vs. uncertain) $\times$ risk-reward relationship (negative vs. positive) $\times$ payoff as predictors).

Did individual differences in learned risk-reward structures help to predict choices of the uncertain alternative in the decision under uncertainty task? As an index of the learned risk-reward structures, we again estimated a risk-reward slope for each participant from the risk-reward task (payoff-dependent estimates with participant as a grouping factor). There was a weak but not credible association between learned risk-reward relationships and the tendency to choose the higher payoff gamble $\left(b_{\text {positive }}=.64, \mathrm{CI}=[-.04,1.33]\right.$, slope $\times$ payoff $\times$ condition interaction in a fixed effects model using the negative condition as baseline, controlling for historical frequencies).

\subsection{Summary}

Experiment 3 revealed that participants could also learn different risk-reward relationships when the probabilities were expressed in the form of epistemic events. The evidence for learning was more pronounced when the relationship was negative than when it was positive, suggesting that the negative association may have been more in line with participants' initial 'priors'. The learned risk-reward relationship impacted subjective probability estimates about the likelihood of the event occurring. Moreover, preferences in subsequent decisions under uncertainty were to some extent environment-dependent. When participants had explicit probability information available in the learning phase-that is, when they learned under risk, choices were impacted in the low-payoff condition as if participants used both their subjective knowledge about the epistemic events and their knowledge of the risk-reward relationship to estimate subjective probabilities. We also found that there were limits to the degree to which participants used the risk-reward relationship: Subsequent choices were not impacted when participants learned under uncertainty.

\section{General discussion}

Ecological structures between risks and rewards that are present in many real-world environments afford people a solution to the problem of unknown probabilities in decisions uncertainty: People can exploit risk-reward structures to infer probabilities from the magnitude of the payoff itself (Pleskac \& Hertwig, 2014). Here, we investigated two requirements of such a solution (1) that people are able to extract the environmental structure and (2) that they use the structure adaptively, as the ecological regularities can and do vary across environments. Our findings from three experiments demonstrate that people can learn risk-reward relationships from the options they experience during preferential choice. Moreover, they learned the relationships without being asked to attend to the structures (incidental, unsupervised learning). Finally, the learned risk-reward relationships can guide the direction of 
A

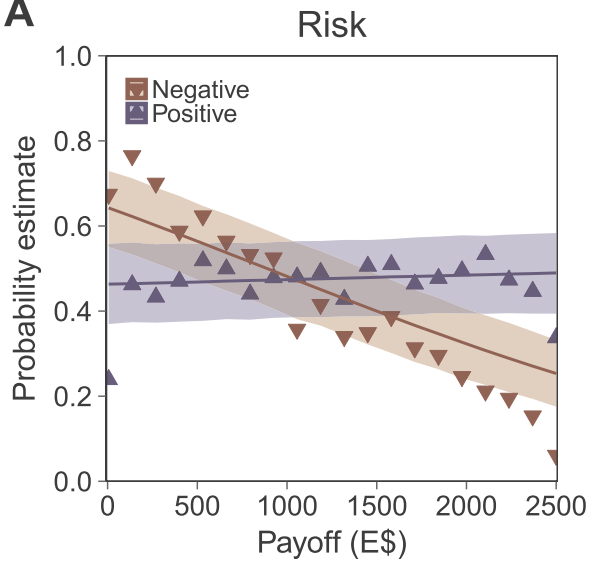

B

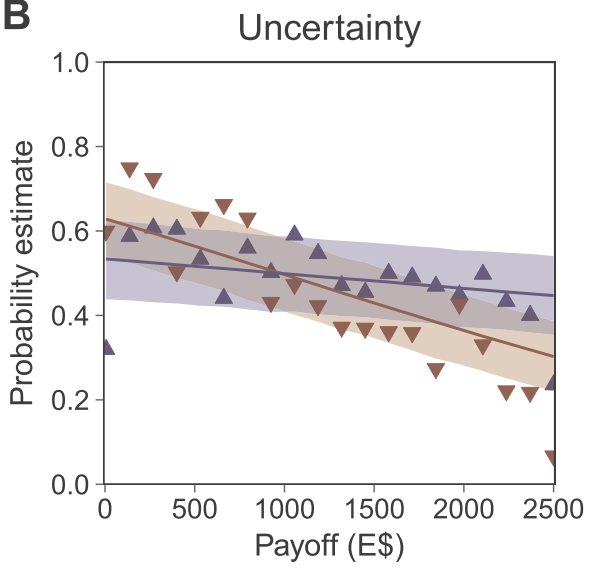

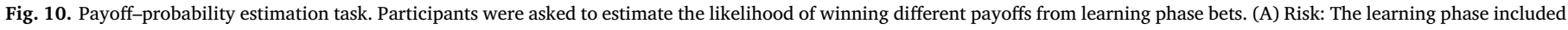

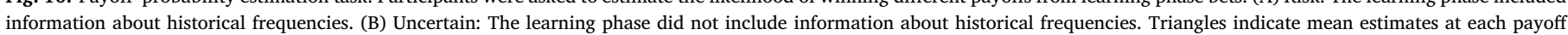
level. Lines (ribbons) indicate the mean (95\% HDIs) of the posterior predictions.

estimates and ultimately impact preferences in decisions made under uncertainty. Next, we discuss our findings in detail and consider their broader implications for adaptive approaches to cognition.

\subsection{Learning risk-reward structures}

Adaptive approaches to cognition seek to understand cognition within the environmental context (Anderson, 1991; Gibson, 1979; Gigerenzer \& Todd, 1999; Marr, 1982; Shepard, 1987; Simon, 1956; Stewart et al., 2006). In the words of Herbert A. Simon (Simon, 1956), "...we might hope to discover, by a careful examination of some of the fundamental structural characteristics of the environment, some further clues as to the nature of the approximating mechanisms used in decision making" (p. 130). Taking this perspective means it is equally important to identify the ecological structures to which a mind may adapt as it is to establish how the mind comes to terms with those ecological structures (Brunswik \& Kamiya, 1953; Simon, 1956).

People can only exploit a risk-reward structure if it has entered the mind. There is good evidence that people are automatic processors of frequency information (a proxy for probabilities) (Hasher \& Zacks, 1979; Zacks, 2002), and distributions of payoffs (Brown et al., 2008; Olivola \& Sagara, 2009; Stewart et al., 2006; Ungemach et al., 2011). The risk-reward relationship is, however, different in that it is the joint distribution of these dimensions across different gambles. Moreover, the central goal in most decision environments is to select the best option(s), and not evaluate them based on their risk-reward relationships. People are neither explicitly informed of those relationships nor do they learn about them from explicit feedback. Instead, it would seem that, if at all, the risk-reward relationship enters the mind via incidental, unsupervised learning (e.g., Brooks, 1978; Love, 2002; Nelson, 1984; Ward \& Scott, 1987; Wattenmaker, 1991). Across three experiments, we showed that participants learn risk-reward relationships from evaluating gambles. Crucially, participants even learned there was no association between risks and rewards, especially when there was no 'local' risk-reward relationship (Experiment 2).

In addition, our results suggest that participants abstracted the relationship as a rule. The strongest support for this conclusion comes from the choice patterns and probability estimates in decisions under uncertainty. Gambles in these tasks did not perfectly map onto learning phase exemplars, yet participants' choices and probability estimates largely resembled the risk-reward rule they had learned previously. Our data cannot, however, pinpoint whether this abstraction occurs during or after encoding (Wattenmaker, 1991). Participants may have used hypotheses about what they know from risk-reward relationships to abstract a rule during encoding (Altmann, Dienes, \& Goode, 1995;
Wattenmaker, 1999), or represented the stimuli as exemplars and retrieved a rule from these exemplars as they needed it (Wattenmaker, 1991).

Our findings point to some general factors that appear to affect how easily risk-reward structures are learned. First, it seems that some risk-reward structures are more difficult to learn than others. Specifically, there was evidence that positive risk-reward structures are more difficult to learn than negative ones: Not all participants picked up on the positive relationship, resulting in weaker positive risk-reward estimates than in the risk-reward structure presented in the learning phase. Since people do not come across positive relationships outside the laboratory very often, they may require more evidence to acquire it. After all, in the real world, there is usually "no free lunch." They may even use prior knowledge and assume that high bonuses are unlikely in the experiment itself. Indeed, as Pleskac and Hertwig (2014) showed, people assume a negative risk-reward relationship without prior learning. Second, risk-reward structures seem to be learned more readily with some response types than with others. A comparison of Experiments 1 and 2 suggests that people are more likely to pick up the risk-reward regularity when pricing gambles one-by-one than when choosing between gambles. It is possible that pricing engages deeper processing than choosing the subjectively better option, leading to better encoding of the relationship. Another reason could be that when people choose between two nondominated gambles for all conditions, a 'local' risk-reward relationship is experienced within the choice pair (i.e., a higher payoff is associated with a lower probability relative to the other gamble). A third factor that hampers learning, as Experiment 3 illustrates, is the level of uncertainty in the choices people learn from. It could be that people need many learning trials to adapt to a new environment under uncertainty. To some extent, such conditions may reflect nonlaboratory environments (where there is both uncertainty, but also continuous learning across many, many "trials").

\subsection{How risk-reward structures impact decisions under uncertainty}

Payoffs and probabilities are the pillars of preference. This makes decision making under uncertainty a vexing problem as one of those pillars-the probabilities-is missing. People are commonly thought to deal with this problem by intuiting subjective probabilities from their knowledge and memory (Fox \& Tversky, 1998; Tversky \& Fox, 1995) or by estimating statistical probabilities from samples of information (Hertwig \& Erev, 2009). Our results support still another ecologically grounded solution, namely, that people estimate the missing probabilities from their immediate choice environments via their learned risk-reward relationships. 
More broadly, these findings fit the general processing assumptions of a risk-reward heuristic. First, people do not seem to memorize exemplars but abstract the risk-reward relationship as a rule. Second, subsequent choice patterns speak for the subsequent use, or retrieval, of this rule, and against algebraic calculation. Taken together, these properties are consistent with the heuristic use of payoff information to estimate probabilities, rather than with the use of more complex methods. However, our experiments also identified some limitations on the use of the risk-reward relationship as a heuristic. For instance, the results of Experiment 3 demonstrate that at least under some circumstances, other information beyond the payoff information is used to infer probabilities about epistemic events. Thus, it is unclear to what extent people use the risk-reward relationship in a noncompensatory manner. This is an important aspect, as ignorining information is sometimes used as a defining characteristic of heuristics (Gigerenzer \& Gaissmaier, 2011).

Regardless, the exploitation of the environmental structure has some immediate implications. One is that, as we have shown, experienced risk-reward environments can create environment-dependent preferences in decisions under uncertainty. In particular, participants in negative risk-reward environments chose the sure thing more often as payoffs increased, but the opposite occurred for participants in positive risk-reward environments. In uncorrelated environments, preferences were less extreme but still tended to track a negative risk-reward environment, perhaps reflecting the pervasiveness of negative risk-reward environments outside the lab. This ecological dependency of preferences brings a new perspective to the proposition that preferences are constructed rather than revealed (Ariely \& Norton, 2008; Lichtenstein \& Slovic, 2006; Payne, Bettman, \& Johnson, 1992; Slovic, 1995). The construction of preferences has typically been understood as the result of people selecting a specific procedure from a larger repertoire of possible strategies to formulate a response (Brandstätter, Gigerenzer, \& Hertwig, 2006; Pachur, Hertwig, \& Brandstätter, 2013; Payne, Bettman, \& Johnson, 1993; Tversky et al., 1988), the dynamic nature of information accumulation that adjusts preferences over time (Busemeyer \& Townsend, 1993), or the ecological (marginal) distribution of monetary payoffs and probabilities (Birnbaum, 1992; Stewart et al., 2006; Stewart et al., 2015; Walasek \& Stewart, 2015). Here, we have shown how experiencing different risk-reward environments can result in substantial, environment-dependent preference shifts in decisions under uncertainty.

These environment-dependent preferences are not indicative of a fallacy, but represent an ecologically rational bet on the structure of the environment. Such a bet is more accurate than ignoring probability information altogether-for example, by using the principle of indifference and assigning equal probabilities to all outcomes (Keynes, 1921). Moreover, our results speak against overtly optimistic estimates that increase as the payoff increases, as implied by the desirability bias (Bar-Hillel \& Budescu, 1995; Edwards, 1954; Krizan \& Windschitl, 2007; Sharot, 2011) or the affect heuristic (Slovic \& Peters, 2006). If anything, participants adapted too little to positive risk-reward environments, perhaps due to the strength and pervasiveness of negative risk-reward environments.

\section{Conclusion}

People often have to make decisions under uncertainty, when probability information is not explicitly stated. In many natural environments, risks and rewards are systematically correlated. This regularity allows people to infer the probability of a payoff from its magnitude, consistent with the use of a risk-reward heuristic. By adjusting their preferences to the respective risk-reward structure, people often manage to make highly adaptive choices under uncertainty.

\section{Author note}

Author contributions were as follows: Conceptualization: C.L., R.H., T.P., \& T.J.P.; Methodology: C.L, T.P., \& T.J.P.; Software: C.L.; Data collection \& curation: C.L.; Formal analysis: C.L.; Writing - Original Draft C.L. \& T.J.P.; Writing - Reviewing \& Editing - C.L., R.H., T.P., \& T.J.P.; Supervision: T.J.P. This research was supported by a fellowship to CL from the MaxNetAging Research School at the Max Planck Institute for Demographic Research in Rostock, Germany.

\section{Conflicts of interest}

None.

\section{Acknowledgements}

We would like to thank Jann Wäscher and Chantal Wysocki for assistance with data collection, and Susannah Goss for editing the manuscript..

\section{Appendix A. Supplementary material}

Supplementary results, figures and screenshots from the experiments referred to in the article can be found in the online version at http://dx.doi.org/10.1016/j.cognition.2018.02.019. All gamble stimuli, data and analysis codes can be retrieved via the Open Science Framework (https://osf.io/m4j79).

\section{References}

Altmann, G., Dienes, Z., \& Goode, A. (1995). Modality independence of implicitly learned grammatical knowledge. Journal of Experimental Psychology: Learning, Memory, and Cognition, 21(4), 899-912.

Anderson, J. R. (1991). The adaptive nature of human categorization. Psychological Review, 98(3), 409-429.

Anderson, R. B., Doherty, M. E., Berg, N. D., \& Friedrich, J. C. (2005). Sample size and the detection of correlation - A signal detection account: Comment on Kareev (2000) and Juslin and Olsson (2005). Psychological Review, 112(1), 268-279. http://dx.doi.org/ 10.1037/0033-295X.112.1.268.

Anderson, J. R., \& Schooler, L. J. (1991). Reflections of the environment in memory. Psychological Science, 2(6), 396-408.

Ariely, D., \& Norton, M. I. (2008). How actions create - Not just reveal - Preferences. Trends in Cognitive Sciences, 12(1), 13-16.

Bar-Hillel, M., \& Budescu, D. (1995). The elusive wishful thinking effect. Thinking \& Reasoning, 1(1), 71-103.

Becker, G. M., DeGroot, M. H., \& Marschak, J. (1964). Measuring utility by a singleresponse sequential method. Behavioral Science, 9(3), 226-232.

Birnbaum, M. H. (1992). Violations of monotonicity and contextual effects in choicebased certainty equivalents. Psychologica l Science, 3(5), 310-314.

Brandstätter, E., Gigerenzer, G., \& Hertwig, R. (2006). The priority heuristic: Making choices without trade-offs. Psycho Logical Review, 113(2), 409-432.

Brooks, D. N. (1978). Nonanalytic concept formation and memory for instances. In E. Rosch, \& B. B. Lloyd (Eds.). Cognition and categorization (pp. 169-215). Hillsdale, NJ: Lawrence Erlbaum Associates.

Brown, G. D., Gardner, J., Oswald, A. J., \& Qian, J. (2008). Does wage rank affect employees well-being? Industrial Relations: A Journal of Economy and Society, 47(3), 355-389.

Brunswik, E. (1955). Representative design and probabilistic theory in a functional psychology. Psychological Review, 62(3), 193-217.

Brunswik, E., \& Kamiya, J. (1953). Ecological cue-validity of 'proximity' and of other gestalt factors. The American Journal of Psychology, 66(1), 20-32.

Busemeyer, J. R., \& Townsend, J. T. (1993). Decision field theory: A dynamic-cognitive approach to decision making in an uncertain environment. Psychological Review, $100(3), 432-459$.

Cleeremans, A., Destrebecqz, A., \& Boyer, M. (1998). Implicit learning: News from the front. Trends in Cognitive Sciences, 2(10), 406-416.

Cooksey, R. W. (1996). Judgment analysis: Theory, methods, and applications. San Diego, CA: Academic Press.

Dulany, D. E., Carlson, R. A., \& Dewey, G. I. (1984). A case of syntactical learning and judgment: How conscious and how abstract? Journal of Experimental Psychology: General, 113(4), 541-555.

Edwards, W. (1954). The theory of decision making. Psychological Bulletin, 51(4), $380-417$.

Edwards, W. (1962). Utility, subjective probability, their interaction, and variance preferences. Conflict Resolution, 6(1), 42-51.

Erickson, M. A., \& Kruschke, J. K. (1998). Rules and exemplars in category learning. 
Journal of Experimental Psychology: General, 127(2), 107-140.

Fox, C. R., \& Clemen, R. T. (2005). Subjective probability assessment in decision analysis: Partition dependence and bias toward the ignorance prior. Management Science, 51(9), 1417-1432.

Fox, C. R., \& Rottenstreich, Y. (2003). Partition priming in judgment under uncertainty. Psychological Science, 14(3), 1 95-200.

Fox, C. R., \& Tversky, A. (1998). A belief-based account of decision under uncertainty. Management Science, 44(7), 879-895.

Frensch, P. A., \& Rünger, D. (2003). Implicit learning. Current Directions in Psychological Science, 12(1), 13-18.

Gelman, A., \& Rubin, D. B. (1992). Inference from iterative simulation using multiple sequences. Statistical Science, 7(4), 457-511.

Gibson, J. (1979). The ecological approach to visual perception. Boston, MA: Houghton Mifflin.

Gigerenzer, G., \& Gaissmaier, W. (2011). Heuristic decision making. Annual Review of Psychology, 62, 451-482.

Gigerenzer, G., Hertwig, R., \& Pachur, T. (2011). Heuristics: The foundations of adaptive behavior. New York, NY: Oxford University Press.

Gigerenzer, G., Hoffrage, U., \& Kleinbölting, H. (1991). Probabilistic mental models: A Brunswikian theory of confidence. Psychological Review, 98(4), 506-528.

Gigerenzer, G., \& Todd, P. the ABC Research Group. (1999). Simple heuristics that make us smart. New York, NY: Oxford University Press.

Hasher, L., \& Zacks, R. T. (1979). Automatic and effortful processes in memory. Journal of Experimental Psychology: General, 108(3), 356-388.

Hasher, L., Zacks, R. T., Rose, K. C., \& Sanft, H. (1987). Truly incidental encoding of frequency information. The American Journal of Psychology, 100(1), 69-91.

Hertwig, R., \& Erev, I. (2009). The description-experience gap in risky choice. Trends in Cognitive Sciences, 13(12), 517-523.

Irwin, F. W. (1953). Stated expectations as functions of probability and desirability of outcomes. Journal of Personality, 21(3), 329-335.

Kareev, Y. (2000). Seven (indeed, plus or minus two) and the detection of correlations. Psychological Review, 107(2), 397-402.

Keynes, J. M. (1921). A treatise on probability. London: MacMillan and Co., Ltd.

Knight, F. H. (1921). Risk, uncertainty, and profit. New York, NY: Sentry Press.

Krizan, Z., \& Windschitl, P. D. (2007). The influence of outcome desirability on optimism. Psychological Bulletin, 133(1), 95-121.

Kruschke, J. K. (2014). Doing Bayesian data analysis: A tutorial with R, JAGS, and Stan (2nd ed.). New York, NY: Academic Press/Elsevier.

Langer, E. J. (1975). The illusion of control. Journal of Personality and Social Psychology, 32(2), 311-328.

Leuker, C., Pleskac, T. J., Pachur, T., \& Hertwig, R. (2017). How the mind exploits risk-reward structures in decisions under risk. In: G. Gunzelmann, A. Howes, T. Tenbrink, \& E. Davelaar (Eds.), Proceedings of the 39th annual conference cognitive science society (pp. 2543-2548). London, UK.

Leuker, C., Pachur, T., Hertwig, R., \& Pleskac, T. J. (2018). Too good to be true? Psychological responses to surprising options in risk-reward environments (Manuscript submitted for publication). Retrieved from http://dx.doi.org/10.17605/ OSF.IO/XAUTN.

Lichtenstein, S., \& Slovic, P. (2006). The construction of preferences. New York, NY: Cambridge University Press.

Love, B. C. (2002). Comparing supervised and unsupervised category learning. Psychonomic Bulletin \& Review, 9(4), 829-835.

Luce, R. D., \& Raiffa, H. (1957). Games and decisions. New York, NY: Dover Publications, Inc.

Marr, D. (1982). Vision: A computational approach. San Francisco, CA: W.H. Freeman and Company.

McFadden, D., Machina, M. J., \& Baron, J. (1999). Rationality for economists? In B. Fischhoff, \& C. F. Manski (Eds.). Elicitation of preferences (pp. 73-110). Dordrecht: Springer.

Nelson, D. G. K. (1984). The effect of intention on what concepts are acquired. Journal of Verbal Learning and Verbal Behavior, 23(6), 734-759.

Newsome, J. (2016). March 7. Man wins \$291 million in lottery, brother wins \$7. CNN.

Olivola, C. Y., \& Oppenheimer, D. M. (2008). Randomness in retrospect: Exploring the interactions between memory and randomness cognition. Psychonomic Bulletin and Review, 15(5), 991-996.

Olivola, C. Y., \& Sagara, N. (2009). Distributions of observed death tolls govern sensitivity to human fatalities. Proceedings of the National Academy of Sciences, 106(52), 22151-22156.

Pachur, T., Hertwig, R., \& Brandstätter, E. (2013). Testing process predictions of models of risky choice: A quantitative mo del comparison approach. Frontiers in Psychology, 4, 646.

Pachur, T., Hertwig, R., \& Steinmann, F. (2012). How do people judge risks: Availability heuristic, affect heuristic, or both? Journal of Experimental Psychology: Applied, 18(3), 314-330.
Payne, J. W., Bettman, J. R., \& Johnson, E. J. (1992). Behavioral decision research: A constructive processing perspective. Annual Review of Psychology, 43(1), 87-131.

Payne, J. W., Bettman, J. R., \& Johnson, E. J. (1993). The adaptive decision maker. New York, NY: Cambridge University Press.

Peirce, J. W. (2007). PsychoPy - Psychophysics software in Python. Journal of Neuroscience Methods, 162(1-2), 8-13.

Perkovic, S., \& Orquin, J. L. (2017). Implicit statistical learning in real-world environments leads to ecologically rational decision making. Psychological Science, 29(1), 34-44.

Pleskac, T. J., \& Hertwig, R. (2014). Ecologically rational choice and the structure of the environment. Journal of Experimental Psychology: General, 143(5), 2000-2019.

Reber, A. S. (1967). Implicit learning of artificial grammars. Journal of Verbal Learning and Verbal Behavior, 6(6), 855-863.

Reber, A. S. (1989). Implicit learning and tacit knowledge. Journal of Experimental Psychology: General, 118(3), 219-235.

Seger, C. A. (1994). Implicit learning. Psychological Bulletin, 115(2), 163-196.

Shanks, D. R., \& St. John, M. F. (1994). Characteristics of dissociable human learning systems. Behavioral an d Brain Sciences, 17(3), 367-395.

Sharot, T. (2011). The optimism bias. Current Biology, 21(23).

Shepard, R. N. (1967). Recognition memory for words, sentences, and pictures. Journal of Verbal Learning and Verbal Behavior, 6(1), 156-163.

Shepard, R. N. (1987). Toward a universal law of generalization for psychological science. Science, 237(4820), 1317-1323.

Simon, H. A. (1956). Rational choice and the structure of the environment. Psychological Review, 63(2), 129-138.

Slovic, P. (1995). The construction of preference. American Psychologist, 50(5), 364-371.

Slovic, P., Finucane, M. L., Peters, E., \& MacGregor, D. G. (2004). Risk as analysis and risk as feelings: Some thoughts about affect, reason, risk, and rationality. Risk Analysis, 24(2), 311-322.

Slovic, P., \& Peters, E. (2006). Risk perception and affect. Current Directions in Psychological Science, 15(6), 322-32 5.

Stan Development Team (2016). RStanArm: Bayesian applied regression modeling via Stan (version 2.9.0-4). (R package version 2.13.1).

Stewart, N., Chater, N., \& Brown, G. D. A. (2006). Decision by sampling. Cognitive Psychology, 53(1), 1-26.

Stewart, N., Reimers, S., \& Harris, A. J. (2015). On the origin of utility, weighting, and discounting functions: How they get their shapes and how to change their shapes. Management Science, 61(3), 687-705.

Todd, P. M., \& Gigerenzer, G. (2007). Environments that make us smart: Ecological rationality. Current Directions in Psychological Science, 16(3), 167-171.

Tversky, A., \& Fox, C. R. (1995). Weighing risk and uncertainty. Psychological Review, 102(2), 269-283.

Tversky, A., \& Kahneman, D. (1986). Rational choice and the framing of decisions. Journal of Business, 59(4), S251-S278.

Tversky, A., \& Kahneman, D. (1992). Advances in prospect theory: Cumulative representation of uncertainty. Journal of Risk and Uncertainty, 5(4), 297-323.

Tversky, A., Sattath, S., \& Slovic, P. (1988). Contingent weighting in judgment and choice. Psychological Review, 95(3), 371-384.

Tversky, A., \& Wakker, P. (1995). Risk attitudes and decision weights. Econometrica, 63(6), 1255-1280.

Ungemach, C., Stewart, N., \& Reimers, S. (2011). How incidental values from the environment affect decisions about money, risk, and delay. Psychological Science, 22(2), $253-260$.

Wakker, P. (2010). Prospect theory for risk and ambiguity. Cambridge, UK: Cambridge University Press.

Walasek, L., \& Stewart, N. (2015). How to make loss aversion disappear and reverse: Tests of the decision by sampling origin of loss aversion. Journal of Experimental Psychology: General, 144(1), 7-11.

Ward, T. B., \& Scott, J. (1987). Analytic and holistic modes of learning family-resemblance concepts. Memory \& Cognition, 15(1), 42-54.

Wattenmaker, W. D. (1991). Learning modes, feature correlations, and memory-based categorization. Journal of Experimental Psychology: Learning, Memory, and Cognition, 17(5), 908-923.

Wattenmaker, W. D. (1999). The influence of prior knowledge in intentional versus incidental concept learning. Memory \& Cognition, 27(4), 685-698.

Whittlesea, B. W. (1987). Preservation of specific experiences in the representation of general knowledge. Journal of Experimental Psychology: Learning, Memory, and Cognition, 13(1), 3-17.

Windschitl, P. D., Smith, A. R., Rose, J. P., \& Krizan, Z. (2010). The desirability bias in predictions: Going optimistic without leaving realism. Organizational Behavior and Human Decision Processes, 111(1), 33-47.

Zacks, R. T. (2002). Frequency processing: A twenty-five year perspective. In P. Sedlmeier, \& T. Betsch (Eds.). Frequency processing and cognition (pp. 21-36). New York, NY: Oxford University Press. 